\title{
Laminar counterflow parallel-plate heat exchangers: Exact and approximate solutions
}

\author{
Marcos Vera ${ }^{\mathrm{a}, *}$, Amable Liñán ${ }^{\mathrm{b}}$ \\ ${ }^{a}$ Dept. de Ingeniería Térmica y de Fluidos, Escuela Politécnica Superior, Universidad Carlos III de Madrid, 28911 Leganés, Spain \\ ${ }^{\mathrm{b}}$ Dept. de Motopropulsión y Termofluidodinámica, E. T. S. I. Aeronáuticos, Universidad Politécnica de Madrid, 28040 Madrid, Spain
}

\begin{abstract}
A B S T R A C T
Multilayered, counterflow, parallel-plate heat exchangers are analyzed numerically and theoretically. The analysis, carried out for constant property fluids, considers a hydrodynamically developed laminar flow and neglects longitudinal conduction both in the fluid and in the plates. The solution for the temperature field involves eigenfunction expansions that can be solved in terms of Whittaker functions using standard symbolic algebra packages, leading to analytical expressions that provide the eigenvalues numerically. It is seen that the approximate solution obtained by retaining the first two modes in the eigenfunction expansion provides an accurate representation for the temperature away from the entrance regions, specially for long heat exchangers, thereby enabling simplified expressions for the wall and bulk temperatures, local heat-transfer rate, overall heat-transfer coefficient, and outlet bulk temperatures. The agreement between the numerical and theoretical results suggests the possibility of using the analytical solutions presented herein as benchmark problems for computational heat-transfer codes.
\end{abstract}

\section{Introduction}

Parallel-plate heat exchangers are widely used in chemical, pharmaceutical, food processing, and many other industrial applications. More recently, they have also found application in a variety of emerging branches of thermal engineering. Thus, they are currently used in miniaturized reaction systems involving heterogeneously catalyzed gas-phase reactions [1], in thermoelectric generators that convert low-grade thermal energy into electrical power [2,3], and in thermoacoustic engines and refrigerators [4]. In addition, they are a key component of many cryogenic systems [5-7].

Progress in the analysis of parallel-plate heat exchangers has been significant in the last decades due to their simple geometry and well established flow conditions [8]. In particular, the analysis of the steady-state laminar heat-transfer between different streams coupled through compatibility conditions at the boundaries constitutes the so-called conjugated Graetz problem [9-11]. Under certain simplifying assumptions - constant property fluids and fully developed laminar flow - the problem becomes linear and is amenable to an elegant solution based on eigenfunction expansions, which in counterflow systems involves sets of positive and negative eigenfunctions associated with sets of positive and negative eigenvalues [11-13].

The aim of this paper is to extend the classical analysis of Nunge and Gill [12] using symbolic algebra to write closed form analytical expressions for the eigenfunctions. This will lead to an exact analytical eigencondition that provides the eigenvalues numerically in a straightforward manner. The theoretical solution for the temperature field will then be written in terms of an infinite eigenfunction expansion. In order to simplify the resulting expressions, we shall derive an approximate solution of the problem retaining only the first two modes in the eigenfunction expansion. This approach provides accurate representations for the temperature field away from the entrance regions, thus enabling simplified expressions for the wall and bulk temperatures, local heat-transfer rate, overall heat-transfer coefficient, Nusselt numbers, and outlet bulk temperatures of both streams, which may be useful for engineering applications even for moderately short heat exchangers. Finally, we shall analyze the singular case of balanced heat exchanger, when zero becomes an algebraically double eigenvalue, and give approximate asymptotic expressions for the relevant case of nearly-balanced heat exchanger.

\section{Problem formulation}

In this paper, we analyze the flow of two constant property Newtonian fluids through a multilayered counterflow parallelplate heat exchanger composed by a relatively large number of 


\section{Nomenclature}

\begin{tabular}{|c|c|}
\hline A & expansion coefficient \\
\hline $\bar{A}$ & $\mathcal{O}(1)$ expansion coefficient in the limit $|\epsilon| \ll 1$ \\
\hline $\bar{B}$ & $\mathcal{O}(1)$ expansion coefficient in the limit $|\epsilon| \ll 1$ \\
\hline$a_{i}$ & channel half-width of fluid $i$ \\
\hline$C_{n}$ & $\begin{array}{l}\text { expansion coefficient corresponding to the } n \text {th eigen- } \\
\text { function }\end{array}$ \\
\hline$c_{i}$ & specific heat of fluid $i$ \\
\hline$f_{n}$ & nth eigenfunction \\
\hline$F_{n, i}$ & $\begin{array}{l}\text { contribution of order } \epsilon^{n} \text { to the oth eigenfunction in the } \\
\text { limit }|\epsilon| \ll 1\end{array}$ \\
\hline $\mathrm{Gz}_{i}$ & Graetz number of fluid $i, \mathrm{Pe}_{i} a_{i} / L$ \\
\hline$h_{i}$ & heat-transfer coefficient of fluid $i$ \\
\hline$I_{i}$ & integral defined in Section (3.4) \\
\hline$k$ & dimensionless parameter, $\left(a_{1} k_{2}\right) /\left(a_{2} k_{1}\right)$ \\
\hline$k_{i}$ & thermal conductivity of fluid $i$ \\
\hline$l_{n}$ & $\begin{array}{l}\text { contribution of order } n \text { to the eigenvalue } \lambda_{0} \text { in the limit } \\
|\epsilon| \ll 1\end{array}$ \\
\hline$L$ & length of the heat exchanger \\
\hline$M$ & Whittaker's function, $M_{\kappa, \mu}(z)$ \\
\hline$m$ & dimensionless parameter, $\left(a_{2} \mathrm{Pe}_{2}\right) /\left(a_{1} \mathrm{Pe}_{1}\right)$ \\
\hline NTU & number of transfer units \\
\hline $\mathrm{Nu}_{i}$ & Nusselt number of fluid $i, h_{i}\left(4 a_{i}\right) / k_{i}$ \\
\hline$\dot{m}_{i}$ & mass flow rate of fluid $i, 2 a_{i} V_{i} \rho_{i}$ \\
\hline $\mathrm{Pe}_{i}$ & Peclet number of fluid $i, 2 a_{i} V_{i} / \alpha_{i}$ \\
\hline $\operatorname{Pr}_{i}$ & Prandtl number of fluid $i, v_{i} / \alpha_{i}$ \\
\hline $\mathcal{R}$ & residual error \\
\hline $\operatorname{Re}_{i}$ & Reynolds number of fluid $i, 2 a_{i} V_{i} / v_{i}$ \\
\hline$R$ & heat-capacity flow rate ratio, $\left(\dot{m}_{i} c_{i}\right)_{\min } /\left(\dot{m}_{i} c_{i}\right)_{\max }$ \\
\hline$T$ & temperature \\
\hline$U$ & overall heat-transfer coefficient \\
\hline$\widetilde{U}$ & dimensionless overall heat-transfer coefficient \\
\hline$u_{i}$ & longitudinal velocity of fluid $i$ \\
\hline$V_{i}$ & average flow velocity of fluid $i$ \\
\hline$W$ & Whittaker's function, $W_{\kappa, \mu}(z)$ \\
\hline$w\left(y_{i}\right)$ & weight function, $(3 / 4)\left(1-y_{i}^{2}\right)$ \\
\hline
\end{tabular}

\section{Greek letters}

$\alpha_{i} \quad$ thermal diffusivity of fluid $i, k_{i} /\left(\rho_{i} c_{i}\right)$

$A_{i} \quad$ bulk temperature offset of fluid $i$ at the inlet

$\epsilon \quad$ small parameter, $1-(m k)^{-1}$

$\varepsilon \quad$ heat exchanger effectiveness

$\Gamma \quad$ gamma function, $\Gamma(z)$

$\kappa \quad$ first argument of Whittaker functions

$\hat{\kappa} \quad$ lumped variable, $m^{1 / 3} k$

$\lambda_{n} \quad n$th eigenvalue

$\mu \quad$ second argument of Whittaker functions

$v \quad$ dimensionless local heat-transfer rate, $\partial \theta_{1} /\left.\partial y_{1}\right|_{y_{1}=1}$

$v_{i} \quad$ kinematic viscosity of fluid $i$

$\rho_{i} \quad$ density of fluid $i$

$\theta_{i} \quad$ dimensionless temperature of fluid $i$

$\Upsilon \quad$ auxiliar function appearing in Eq. (31)

$\xi$ dimensionless longitudinal coordinate

$\Omega_{n} \quad$ coefficient appearing in Eq. (34)

\section{Subscripts}

$\begin{array}{ll}i & \text { subscript used indistinctly for fluids } 1 \text { and } 2 \\ \text { in } & \text { inlet } \\ L & \text { length of the heat exchanger } \\ m & \text { bulk, or mixing-cup, temperature } \\ n & \text { corresponding to the } n \text {th eigenvalue/eigenfunction } \\ \text { out } & \text { outlet } \\ w & \text { heat-exchanging wall }\end{array}$

\section{Superscripts}

(0) zeroth-order two-term solution

(1) first-order two-term solution

[N] $2(N+1)$-term exact truncated solution channels separated by thermally thin plates. The conducting plates allow the exchange of heat through a section of length $L$, presenting insulated sections at both ends of the heat exchange section where no heat-transfer is allowed [14]. In the configuration considered here, the two fluids, denoted by 1 and 2 (the subscript $i=1,2$ will be used indistinctly for both fluids), flow in opposite directions in adjacent channels. Then, if the characteristic dimension of the heat exchanger is large compared with the channel widths, $a_{i}$, the temperature field, as seen with this scale, appears as periodic in the transverse direction, with period $2\left(a_{1}+a_{2}\right)$. Accordingly, when describing the temperature field in the unitary cell of the heat exchanger we can use symmetry boundary conditions at the channel symmetry planes, where no energy can be transferred.

Fig. 1 shows a sketch of the physical model under study, where $X$ denotes the longitudinal coordinate measured from the inlet of fluid 1 , and $Y_{i}$ denotes the transverse coordinate measured from the symmetry plane of channel $i$. The Reynolds numbers of the flow in the channels, $\operatorname{Re}_{i}=2 a_{i} V_{i} / v_{i}$, based on the average flow velocity, $V_{i}$, and kinematic viscosity, $v_{i}$, of fluid $i$, will be considered to be sufficiently small for the flow to be laminar [15]. In addition, the Prandtl number, $\operatorname{Pr}_{i}=v_{i} / \alpha_{i}$, defined in terms of the thermal diffusivity, $\alpha_{i}$, of fluid $i$, is assumed to be large compared to unity, so that the thermal entry length is large compared to the hydrodynamic entry length, a good approximation for most non-metallic liquids. Then, the flow will be assumed to be a fully developed Poiseuille flow and, due to the constant property assumption, independent of the temperature field, $u_{i}\left(Y_{i}\right)= \pm(3 / 2) V_{i}\left[1-\left(Y_{i} / a_{i}\right)^{2}\right]$, where the plus sign holds for fluid 1 and the minus for fluid 2 .

In addition, we shall assume here that the Peclet numbers of the flow in the channels, $\operatorname{Pe}_{i}=\operatorname{Re}_{i} \operatorname{Pr}_{i}=2 a_{i} V_{i} / \alpha_{i}$, are both large compared to unity. In this case, axial heat conduction in the fluids is confined to small regions located at the end sections of the heat exchanger, close to the heat conducting plates, whose characteristic size, of order $\left(\alpha_{i} a_{i} / V_{i}\right)^{1 / 2}=\operatorname{Pe}_{i}^{-1 / 2} a_{i}$, is small compared to $a_{i}$ when $\mathrm{Pe}_{i} \gg 1$.

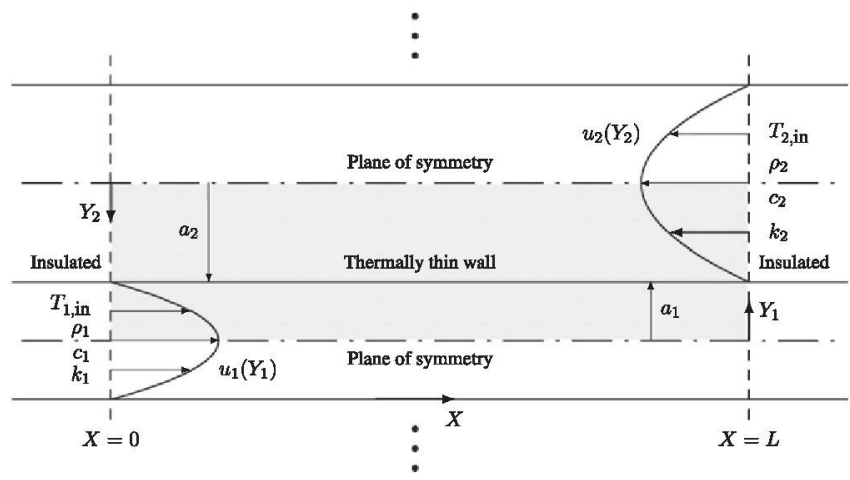

Fig. 1. Unitary cell of the idealized counterflow parallel-plate heat exchanger showing the coordinate system, the velocity field, and the physical properties of fluids 1 and 2 . The domain under study is shaded gray. 
In this limiting case, we can neglect axial conduction in the fluid in first approximation, and thus we will assume that the inlet temperature of fluid $i$ is uniform and equal to $T_{i, \text { in }}$. Previous analyses of counterflow heat exchangers have concluded that this is a good approximation for Peclet numbers of order 100 or more $[16,17]$.

To write the problem in non-dimensional form, we introduce the dimensionless variables $\xi=X /\left(\mathrm{Pe}_{1} a_{1}\right), y_{i}=Y_{i} / a_{i}$, and $\theta_{i}=$ $\left(T_{i}-T_{1, \text { in }}\right) /\left(T_{2, \text { in }}-T_{1, \text { in }}\right)$. Then, under the assumptions stated above, the energy equation reduces, in first approximation, to a balance between axial convection and transverse conduction

$$
\begin{aligned}
& \frac{3}{4}\left(1-y_{1}^{2}\right) \frac{\partial \theta_{1}}{\partial \xi}=\frac{\partial^{2} \theta_{1}}{\partial y_{1}^{2}}, \\
& -m \frac{3}{4}\left(1-y_{2}^{2}\right) \frac{\partial \theta_{2}}{\partial \xi}=\frac{\partial^{2} \theta_{2}}{\partial y_{2}^{2}},
\end{aligned}
$$

in $0<\xi<\xi_{L}, 0<y_{i}<1$, where the dimensionless parameters

$m=\frac{a_{2}}{a_{1}} \frac{\mathrm{Pe}_{2}}{\mathrm{Pe}_{1}}$ and $\xi_{L}=\frac{L}{a_{1}} \frac{1}{\mathrm{Pe}_{1}}$,

are both assumed to be of order unity. Note that $m=\mathrm{Gz}_{2} / \mathrm{Gz}_{1}$ can also be viewed as the ratio of the Graetz number of both streams, defined as $\mathrm{Gz}_{i}=\mathrm{Pe}_{i} a_{i} / L$. Note also that the dimensionless coordinate, $\xi$, is defined here using the thermal entry length of fluid 1 as length scale. As a consequence, the heat exchanger length shows up in the dimensionless parameter $\xi_{L}$. Other investigators prefer to use the heat exchanger length as length scale, in which case $\xi_{L}$ would appear through the Graetz numbers $\mathrm{Gz}_{i}$ arising in the convective terms. Note also that due to the existing counterflow pattern, the mathematical problem is elliptical rather than parabolic even though longitudinal heat conduction effects are not taken into account.

The above equations have to be integrated with the boundary conditions at the channel symmetry planes

$\frac{\partial \theta_{1}}{\partial y_{1}}=\frac{\partial \theta_{2}}{\partial y_{2}}=0 \quad$ at $\quad 0<\xi<\xi_{L}, \quad y_{i}=0$

at the heat conducting wall

$$
\begin{gathered}
\theta_{1}=\theta_{2} \equiv \theta_{w}(\xi) \\
\frac{\partial \theta_{1}}{\partial y_{1}}=-k \frac{\partial \theta_{2}}{\partial y_{2}} \equiv v(\xi)
\end{gathered} \quad \text { at } \quad 0<\xi<\xi_{L}, \quad y_{i}=1,
$$

and at the inlet sections

$\theta_{1}=\theta_{1, \text { in }}=0 \quad$ at $\quad \xi=0, \quad 0 \leqslant y_{1}<1$,

$\theta_{2}=\theta_{2, \text { in }}=1$ at $\xi=\xi_{L}, \quad 0 \leqslant y_{2}<1$.

In the thermally thin wall regime considered here the absence of longitudinal heat conduction effects for $\mathrm{Pe}_{i} \gg 1$ implies that the wall temperature at both ends of the heat exchanging region, $\theta_{w}(0)$ and $\theta_{w}\left(\xi_{L}\right)$, is imposed by the fluid entering the exchanger in that section, so that we may anticipate that $\theta_{w}(0)=0$ and $\theta_{w}\left(\xi_{L}\right)=1$, as will be later confirmed by the numerical solutions discussed in Section 6 .

Note that the condition of continuity of heat fluxes at the heat conducting wall (5) introduces the additional parameter

$k=\frac{a_{1}}{a_{2}} \frac{k_{2}}{k_{1}}$

However, one may alternatively choose the ratio of the heatcapacity flow rates $m k=\left(\dot{m}_{2} c_{2}\right) /\left(\dot{m}_{1} c_{1}\right)$ as main parameter together with either $m$ or $k$ - see the analysis for small values of $1-(m k)^{-1}$ and arbitrary values of $k$ given in Appendix A. It is important to note that while the heat-capacity flow rate ratio is the most relevant dimensionless parameter in lumped methods, such as the LMTD or $\varepsilon$-NTU methods, the differential analysis of laminar counterflow heat exchangers always requires the use of two independent dimensionless parameters, such as $m$ and $k$.
The solution to this problem provides the temperature field, $\theta_{i}\left(\xi, y_{i}\right)$, as well as the plate temperature, $\theta_{w}(\xi)$, for given values of $m, k$, and $\xi_{L}$. It also provides the local heat-transfer rate, $v(\xi)$, defined in (5), which represents the dimensionless heat flux from fluid 2 to fluid 1 , and the outlet temperature profiles

$\theta_{1}=\theta_{1, \text { out }}\left(y_{1}\right)$ at $\xi=\xi_{L}, \quad 0 \leqslant y_{1}<1$,

$\theta_{2}=\theta_{2, \text { out }}\left(y_{2}\right)$ at $\xi=0, \quad 0 \leqslant y_{2}<1$.

Another interesting result is the distribution of the bulk - or mixing-cup - temperatures of both streams

$\theta_{m i}(\xi)=\int_{0}^{1} \frac{3}{2}\left(1-y_{i}^{2}\right) \theta_{i}\left(\xi, y_{i}\right) d y_{i}$

defined as the uniform temperature that would eventually be attained if the fluid at a particular section $\xi$ was allowed to evolve adiabatically. The main outcome of the analysis is, however, the values of the outlet bulk temperatures of both streams, $\theta_{m 1 \text {,out }} \equiv \theta_{m 1}\left(\xi_{L}\right)$ and $\theta_{m 2 \text {,out }} \equiv \theta_{m 2}(0)$, closely related to the heat exchanger effectiveness, as will be seen below.

Integrating (1) and (2) across the channel and using (5) we have

$\frac{d\left(\theta_{m 2}-\theta_{m 1}\right)}{d \xi}=\left[(m k)^{-1}-1\right] 2 v(\xi)$,

which highlights the role of the heat-capacity flow rate ratio, $m k$, in the axial evolution of the bulk temperature difference. Thus, if the heat-capacity flow rates of both streams are equal the bulk temperature difference, $\theta_{m 2}-\theta_{m 1}(>0)$, takes a constant value along the heat exchanger, while if the heat-capacity flow rate of fluid 2 is larger (smaller) than that of fluid $1, m k>1(<1)$, the bulk temperature difference decreases (increases) with $\xi$. Alternatively, from (1), (2), and (5) we may also write

$\frac{d\left(m k \theta_{m 2}-\theta_{m 1}\right)}{d \xi}=0$,

concluding that the net convective thermal energy flux across any longitudinal cross-section of the heat exchanger must be constant, as follows from and integral energy balance. Using the boundary conditions at both ends of the heat exchanger this equation can be readily integrated to give $m k \theta_{m 2}(\xi)-\theta_{m 1}(\xi)=m k \theta_{m 2 \text {,out }}=m k-\theta_{m 1 \text {,out, }}$ which relates the outlet bulk temperatures of both streams according to

$\theta_{m 1, \text { out }}=m k\left(1-\theta_{m 2, \text { out }}\right), \quad \theta_{m 2, \text { out }}=1-(m k)^{-1} \theta_{m 1, \text { out }}$.

The classical way to attack this problem is to use approximate expressions for the local heat-transfer rate $v(\xi)$ in terms of the bulk temperature difference, $\theta_{m 2}-\theta_{m 1}$, so as to integrate (12) and determine the outlet bulk temperatures, $\theta_{\text {mi,out }}$. A customary choice is to assume the local heat-transfer rate to be proportional to the local bulk temperature difference

$v(\xi)=\widetilde{U}(\xi)\left(\theta_{m 2}-\theta_{m 1}\right)$,

introducing the dimensionless overall heat-transfer coefficient $\widetilde{U}(\xi)$. For preliminary design purposes, a useful approximation is to take $\widetilde{U}(\xi)$ equal to a constant, average value, $\bar{U}$, along the heat exchanger. This approach has led to the development of lumped methods, such as the traditional LMTD method, used for heat exchanger design together with $F$ correction factor charts, or the $\varepsilon$-NTU method, used for performance calculations $[18,19]$. As a further step, mixed lumped-differential methods have also been developed [20]. Because of the simplifying assumptions underlying these approximate methods, they are unable to give a detailed picture of the temperature distribution inside the exchanger, particularly in the thermal entrance region, where a significant fraction of the total heat may be transferred - at least in short-length heat exchangers. 
An alternative way is the use of numerical methods to obtain the solution by force brute for every possible combination of the governing parameters. However, in order to unveil parametric trends or to determine the structure of the solution in certain limiting cases, the numerical approach must go hand-in-hand with theoretical analysis. Nonetheless, the analytical solution of the problem, to be derived below, is in general prohibitively complicated for all but the simplest configurations, as the one considered here.

\section{Theoretical analysis by separation of variables}

\subsection{General formulation}

Following standard practice, the solution of the problem will be sought in the form of eigenfunction expansions. Assuming separation of variables of the form $\theta_{i}\left(\xi, y_{i}\right)=\phi_{n}(\xi) f_{n, i}\left(y_{i}\right)$ leads to $\phi_{n}(\xi)=e^{-\lambda_{n} \xi}$ in $0 \leqslant \xi \leqslant \xi_{L}$. Thus, the solution may be written as

$\theta_{i}\left(\xi, y_{i}\right)=A+\sum_{n=-\infty}^{+\infty} C_{n} e^{-\lambda_{n} \xi} f_{n, i}\left(y_{i}\right)$,

where the undetermined constant $A$ arises due to the invariance of Eqs. (1) and (2) under arbitrary shifts in temperature. Note that this constant could also be viewed as an additional eigenfunction, the corresponding eigenvalue being identically zero [12].

Satisfaction of the inlet boundary conditions (6) and (7) at both ends of the heat exchanger forces us to use a set of both positive $(n>0)$ and negative $(n<0)$ eigenvalues, $\lambda_{n}$, and their corresponding eigenfunctions, $f_{n, i}\left(y_{i}\right)$ [12]. Hereafter, we shall denote with $n=0$ the eigenvalue with the lowest absolute value. This eigenvalue, which determines the behavior of the solution away from the entrance regions, is anticipated to be positive for $m k>1$ and negative for $m k<1$, according to the discussion following Eq. (12).

The singular case $m k=1$, when 0 is an algebraically double eigenvalue, is treated in detail in Appendix A. Specifically, as $1-(m k)^{-1}=\epsilon \rightarrow 0$ the eigenfunction $f_{0, i}\left(y_{i}\right)$ approaches unity while the coefficient $C_{0}$ diverges as $\epsilon^{-1}$. This singular behavior must be counterbalanced by $A$, which also diverges as $\epsilon^{-1}$, so that $\bar{A}=A+C_{0}$ remains bounded, eventually leading to the alternative series expansion given in Eq. (72).

Use of expansion (16) in Eqs. (1) and (2) leads to the following problem for the $n$th eigenfunction

$-\lambda_{n} \frac{3}{4}\left(1-y_{1}^{2}\right) f_{n, 1}=\frac{d^{2} f_{n, 1}}{d y_{1}^{2}}$,

$\lambda_{n} m \frac{3}{4}\left(1-y_{2}^{2}\right) f_{n, 2}=\frac{d^{2} f_{n, 2}}{d y_{2}^{2}}$,

for $0<y_{i}<1$, to be integrated with the boundary conditions

$$
\begin{aligned}
& \frac{d f_{n, 1}}{d y_{1}}=\frac{d f_{n, 2}}{d y_{2}}=0 \quad \text { at } y_{i}=0, \\
& \frac{d f_{n, 1}}{d y_{1}}=-k \frac{d f_{n, 2}}{d y_{2}} \equiv f_{n, 1}^{\prime}(1) \text { at } y_{i}=1,
\end{aligned}
$$

derived from (4) and (5), respectively, where the value, $f_{n, 1}^{\prime}(1)$, of $d f_{n, 1} / d y_{1}$ at $y_{1}=1$ is to be obtained as part of the solution. Due to the linearity of the problem we must also specify a normalization condition. Thus, without loss of generality, we set

$f_{n, i}=1$ at $y_{i}=1$.

\subsection{Determination of the eigenfunctions}

The analytical solution to the problem stated in (17)-(21) can be obtained using standard symbolic mathematical software packages, such as Mathematica or Maple, $f_{n, i}=\frac{1}{\sqrt{y_{i}}}\left\{\frac{2 \sqrt{\pi} M_{\kappa_{n, i \frac{1}{4}}}\left(4 \kappa_{n, i} y_{i}^{2}\right)+A_{n, i} W_{\kappa_{n, i}, \frac{1}{4}}\left(4 \kappa_{n, i} y_{i}^{2}\right)}{2 \sqrt{\pi} M_{\kappa_{n, i, 4}}\left(4 \kappa_{n, i}\right)+A_{n, i} W_{\kappa_{n, i}, \frac{1}{4}}\left(4 \kappa_{n, i}\right)}\right\}$,

where $M_{\kappa, \mu}(z)$ and $W_{\kappa, \mu}(z)$ denote the Whittaker functions [21,22], and we introduce the complex dimensionless parameters

$A_{n, i}=\Gamma\left(\frac{1}{4}-\kappa_{n, i}\right), \quad \kappa_{n, 1}=\frac{\sqrt{3 \lambda_{n}}}{8}, \quad \kappa_{n, 2}=\mathbf{i} \frac{\sqrt{3 m \lambda_{n}}}{8}$,

where $\Gamma(z)$ is the Gamma function and $\mathbf{i}$ the imaginary unit. Note that the eigenvalue $\lambda_{n}$ appears both in the argument and in the first index of the Whittaker functions through $\kappa_{n, i}$ [23]. Note that although $f_{n, i}$ could also be written in terms of confluent hypergeometric, or parabolic cylinder functions, Whittaker functions provide a more compact representation.

\subsection{Determination of the eigenvalues}

In order to determine the eigenvalues, we apply symbolic derivation to (22) and evaluate the derivatives at the wall

$$
\begin{aligned}
& \left.f_{n, i}^{\prime}\left(1 ; m, \lambda_{n}\right) \equiv \frac{d f_{n, i}}{d y_{i}}\right|_{y_{i}=1} \\
& \quad=-\frac{1}{2}+2\left\{\kappa_{n, i}+\frac{2 \sqrt{\pi}\left(3 / 4+\kappa_{n, i}\right) M_{\kappa_{n, i}+1, \frac{17}{4}}\left(4 \kappa_{n, i}\right)-A_{n, i} W_{\kappa_{n, i}+1, \frac{1}{4}}\left(4 \kappa_{n, i}\right)}{\left.2 \sqrt{\pi} M_{\kappa_{n, i, \frac{1}{4}}\left(4 \kappa_{n, i}\right)+A_{n, i} W_{\kappa_{n, i}, \frac{1}{4}}\left(4 \kappa_{n, i}\right)}\right\},}\right.
\end{aligned}
$$

where $A_{n, i}$ and $\kappa_{n, i}$ are given in terms of $m$ and $\lambda_{n}$ by (23). Then, using (24) in (20) we arrive to the transcendental equation

$f_{n, 1}^{\prime}\left(1 ; m, \lambda_{n}\right)+k f_{n, 2}^{\prime}\left(1 ; m, \lambda_{n}\right)=0$,

which restricts the values of $\lambda_{n}$ to an infinite numerable set of eigenvalues, which could be obtained numerically for given values of $m$ and $k$. However, one may alternatively write (25) as $k=-f_{n, 1}^{\prime}\left(1 ; m, \lambda_{n}\right) / f_{n, 2}^{\prime}\left(1 ; m, \lambda_{n}\right)$, which provides $k$ explicitly in terms of $m$ and $\lambda_{n}$. This procedure was used to generate the contour plots of $\lambda_{0}, \lambda_{ \pm 1}$ and $\lambda_{ \pm 2}$ shown in Fig. 2. The results indicate that the positive eigenvalues are weakly dependent on both $m$ and $k$, while the negative eigenvalues show a strong dependence on $m$. This is due to the mathematical symmetries of the problem, $1 \leftrightarrow 2, m \leftrightarrow m^{-1}$, $k \leftrightarrow k^{-1}, n \leftrightarrow-n$, which allow us to write

$f_{-n, i}^{\prime}(1 ; m, k)=-k f_{n, i}^{\prime}\left(1 ; m^{-1}, k^{-1}\right)$,

$\lambda_{-n}(m, k)=-m^{-1} \lambda_{n}\left(m^{-1}, k^{-1}\right)$,

showing that if $\lambda_{n}$ is roughly constant then $\lambda_{-n}(m, k)$ must grow linearly with $m^{-1}$. Note that hereafter we take $k$ instead of $\lambda_{n}$ as second independent parameter of $f_{-n, i}^{\prime}(1)$, writing $f_{i, 1}^{\prime}(1 ; m, k) \equiv f_{i, 1}^{\prime}$ $\left(1 ; m, \lambda_{n}(m, k)\right)$.

Table 1 shows the eigenvalues $\lambda_{n}$ obtained numerically for $n=0, \pm 1, \ldots, \pm 4$ and selected values of $m$ and $k$. As starting point for the calculations, we used the asymptotic expression

$\lambda_{n}^{0} \simeq \frac{64}{3}\left\{n+\frac{1}{3}+\frac{1}{2 \pi} \arctan \left[\frac{\hat{\kappa}^{2}-2 \hat{\kappa}-2}{3^{1 / 2} \hat{\kappa}(\hat{\kappa}+2)}\right]\right\}^{2}$ as $n \rightarrow \infty$,

with $\hat{\kappa}=m^{1 / 3} k$, obtained by applying symbolic derivation to the Airy asymptotic form of Whittaker functions [24, pp. 31] and substituting the resulting expressions in (25). Eqs. (27) and (28) provide asymptotic expressions for both positive and negative eigenvalues, yielding relative errors of order $10^{-2}$ for $n=1$, and less than $10^{-3}$ for $n \geqslant 5$, as seen in Fig. 2. It is worth noting that higher-order eigenvalues depend solely on the lumped parameter $m^{1 / 3} k$, which must therefore be the relevant parameter in the thermal entrance regions.

The eigenvalues reported in the first three columns of Table 1 were used to calculate the eigenfunctions $f_{n, i}$ shown in Fig. 3. As can be seen, the eigenfunction corresponding to $\lambda_{n}$ has $|n|$ zeros, 

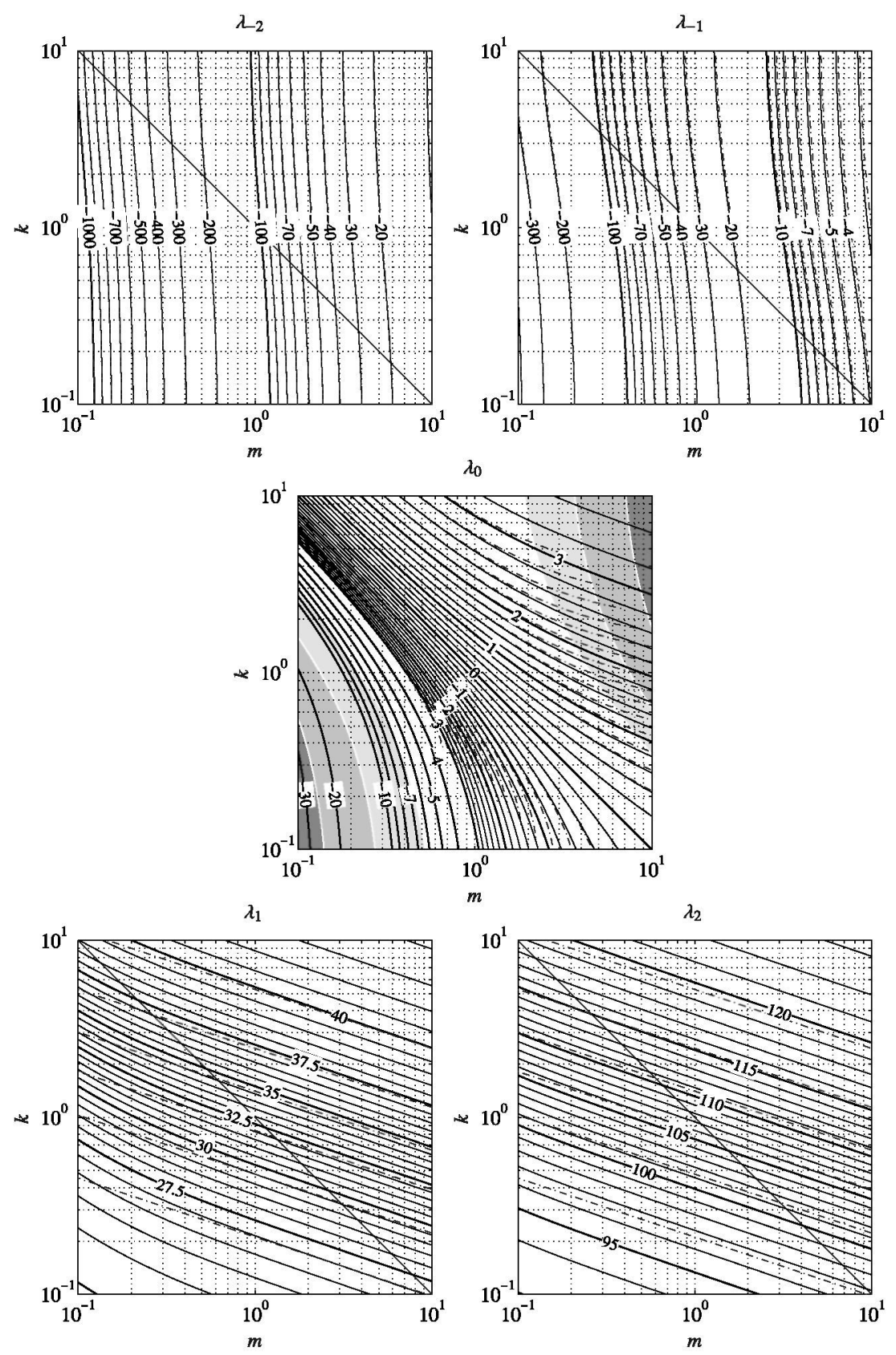

$\lambda_{0}$ 
Table 1

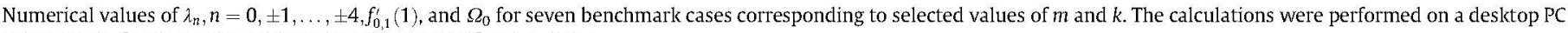
using Maple floating-point arithmetics with 32 significative digits.

\begin{tabular}{|c|c|c|c|c|c|c|c|}
\hline$m$ & 1 & 1 & 1 & 2 & 2 & 2 & 4 \\
\hline$k$ & 1 & 2 & 4 & $1 / 2$ & 1 & 2 & 1 \\
\hline$f_{0,1}^{\prime}(1)$ & 0 & -1.02792579216 & -3.07440396324 & 0 & -0.699849499134 & -2.13170956706 & -1.34250544459 \\
\hline$\Omega_{0}$ & -1 & -1.73652001021 & -4.28178913871 & -1 & -1.37606720686 & -2.80236111420 & -1.82333585522 \\
\hline$\lambda_{0}$ & 0 & 1.35767399721 & 2.38755152930 & 0 & 1.03864103603 & 2.04627989891 & 1.60263301966 \\
\hline$\lambda_{-1}$ & -32.9405413295 & -29.6865385764 & -27.3759045317 & -17.5981961833 & -15.8396694677 & -14.3569208547 & -7.58563339299 \\
\hline$\lambda_{+1}$ & 32.9405413295 & 36.3947820526 & 39.1172555519 & 30.7375596746 & 34.1760757889 & 37.4533370731 & 35.3730746020 \\
\hline$\lambda_{-2}$ & -107.539380992 & -101.679709099 & -97.4057670022 & -55.8024248147 & -52.7002033421 & -49.9969826950 & -25.8321309693 \\
\hline$\lambda+2$ & 107.539380992 & 113.599559702 & 118.285802413 & 103.550411116 & 109.685299476 & 115.402488317 & 111.764208390 \\
\hline$\lambda_{-3}$ & -224.817897962 & -216.358979131 & -210.128124336 & -115.331226866 & -110.883954394 & -106.964939667 & -54.7073490801 \\
\hline$\lambda+3$ & 224.817897962 & 233.479390839 & 240.126795367 & 219.051087913 & 227.875334746 & 236.027201505 & 230.833608675 \\
\hline$\lambda_{-4}$ & -384.772710429 & -373.717983371 & -365.532581533 & -196.196863170 & -190.406244834 & -185.273008426 & -94.2490037567 \\
\hline$\lambda+4$ & 384.772710429 & 396.032130758 & 404.638390387 & 377.229925459 & 388.739945860 & 399.323523773 & 392.575959793 \\
\hline
\end{tabular}
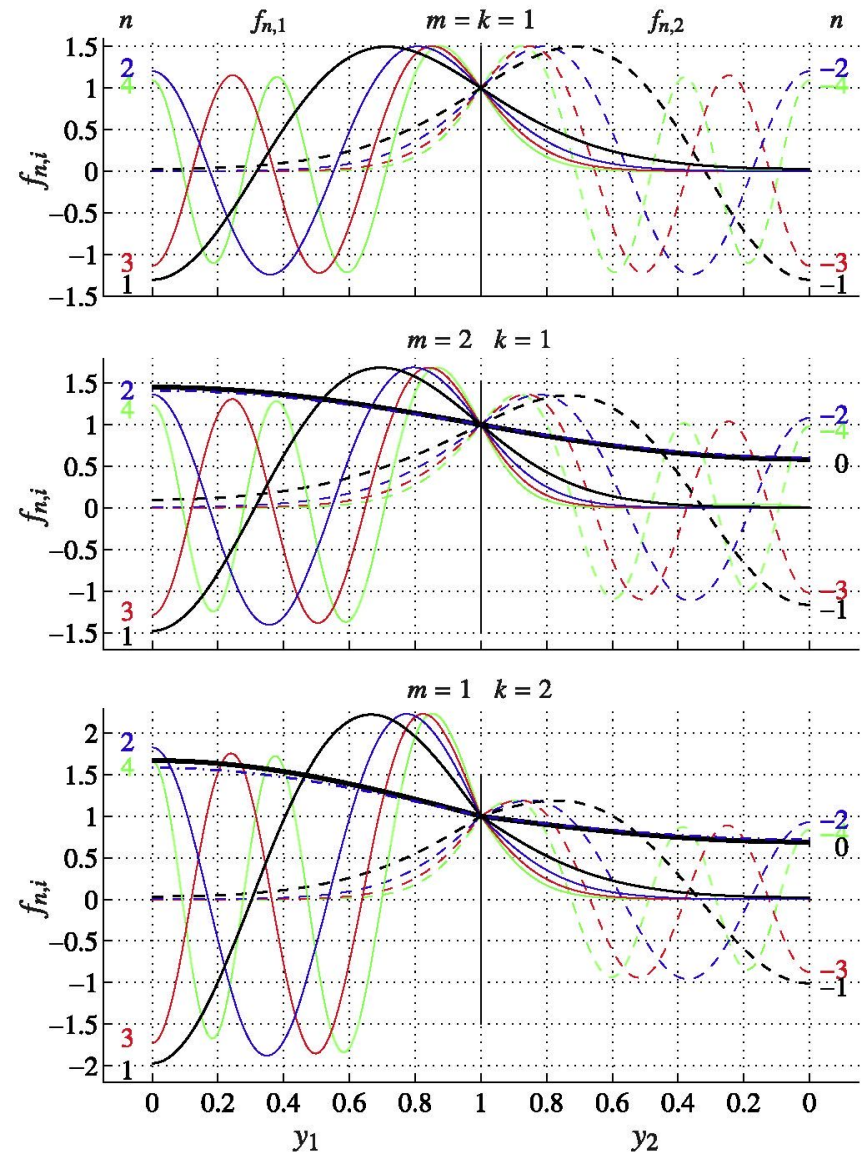

Fig. 3. Eigenfunctions $f_{n, i}$ for $n=0, \pm 1, \ldots, \pm 4$ and selected values of $m$ and $k$. The eigenfunction $f_{0, i}$ is not shown in the upper plot, where $f_{0, i} \equiv 1$ (see Appendix A for details). Dash-dotted lines (middle and lower plots): approximate form of $f_{0, i}$ given by (69) together with (73)-(80) for $\left|1-(m k)^{-1}\right|=|\epsilon| \ll 1$.

constant $A$ in (16) could therefore be viewed as another eigenfunction, the corresponding eigenvalue being identically zero.

\subsection{Determination of the expansion coefficients}

The orthogonality condition for the eigenfunctions may be used together with the inlet conditions (6) and (7) to calculate the expansion coefficients $A$ and $C_{n}[12,13]$ (see Appendix B for details). Omitting the lengthy algebra, this procedure leads, for $m k \neq 1$, to the following infinite set of linear equations $m k=A(1+m k)-\sum_{q=-\infty}^{+\infty} C_{q}\left(1+e^{-\lambda_{q} \xi_{L}}\right) 2 \lambda_{q}^{-1} f_{q, 1}^{\prime}(1)$,

and

$$
\begin{aligned}
e^{\lambda_{n} \xi_{L}} \lambda_{n}^{-1} f_{n, 1}^{\prime}(1)= & A\left[1+e^{\lambda_{n} \xi_{L}}\right] \lambda_{n}^{-1} f_{n, 1}^{\prime}(1)+C_{n} \Omega_{n}+\sum_{\substack{q=-\infty \\
q \neq n}}^{+\infty} C_{q}[1 \\
& \left.+e^{\left(\lambda_{n}-\lambda_{q}\right) \xi_{L}}\right]\left[\frac{f_{n, 1}^{\prime}(1)-f_{q, 1}^{\prime}(1)}{\lambda_{n}-\lambda_{q}}\right]
\end{aligned}
$$

for $n=0, \pm 1, \pm 2, \ldots$, where the coefficient

$$
\Omega_{n}=\frac{\partial}{\partial \lambda_{n}}\left[f_{n, 1}^{\prime}(1)-k f_{n, 2}^{\prime}(1)\right]
$$

must be evaluated numerically, since the derivatives of $f_{n, i}^{\prime}(1)$ with respect to $\lambda_{n}$ cannot be written, in general, in analytical form. Table 1 shows the value of $\Omega_{0}$ thus obtained for selected values of $m$ and $k$. Values of $\Omega_{n}$ can be obtained similarly for $n>0$, while for $n<0$ one may use the symmetry relation $\Omega_{-n}(m, k)=m k \Omega_{n}\left(m^{-1}, k^{-1}\right)$.

The infinite system (32) and (33) must be truncated to a finite number of terms in order to get a numerical solution, with the particularity that the addition of new terms to the solution has an effect on the preceding expansion coefficients, which cannot be calculated successively as typically occurs in single-stream or co-current flow system [12,13]. Moreover, the convergence of the series becomes very slow at small axial distances, where a large number of terms is required for an accurate description of the solution [11,12]. Accordingly, as the inlets are approached the calculation becomes tedious and the numerical solution may not even converge.

In addition, the truncation process introduces additional mathematical difficulties that hinder its widespread practical application. Specifically, for fixed values of $m$ and $k-$ and therefore of $\lambda_{n}, f_{n, i}^{\prime}(1)$ and $\Omega_{n}$ - the truncated system becomes singular for well definite heat exchanger lengths, leading to physically uncorrect solutions for neighboring values of $\xi_{L}$. These singularities show up quite clearly in Fig. 4, where the outlet bulk temperature provided by the truncated solution

$\theta_{m 1, \text { out }}^{[N]}\left(\xi_{L}\right)=A-\sum_{n=-N}^{n=+N} 2 C_{n} e^{-\lambda_{n} \xi_{L}} \lambda_{n}^{-1} f_{n, 1}^{\prime}(1)$,

is plotted as a function of $\xi_{L}$ for $N=0$ (two-terms), $N=1$ (fourterms), and $N=20$ (42 terms), included here as an example of a fully converged solution. Other interesting feature of the truncated solution seen in the insets (a1) and (a2) of Fig. 4 is that the outlet bulk temperature of fluid 1 does not vanish as $\xi_{L} \rightarrow 0$. Instead, we have

$\Delta_{1}^{[N]} \equiv \lim _{\xi_{L} \rightarrow 0} \theta_{m 1, \text { out }}^{[N]}\left(\xi_{L}\right)>0$, with $\lim _{N \rightarrow \infty} \Delta_{1}^{[N]}=0$. 


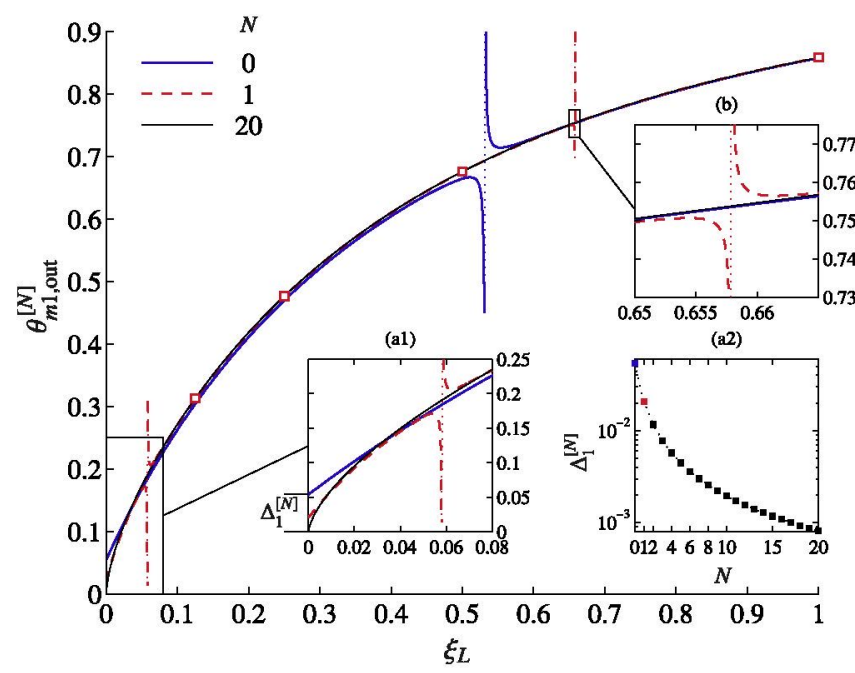

Fig. 4. Variation of the outlet bulk temperature of fluid 1 with the heat exchanger length corresponding to $m=1$ and $k=2$ given by the truncated exact solution, Eq. (35), for $N=0$ (two-term solution), $N=1$ (four-term solution), and $N=20$ (42 term solution), and by the numerical integration of the problem ( $\square$ ). Note the singularities in insets (a1) and (b) corresponding to $N=1$, and the definition of $\Delta_{1}^{[\mathrm{NT}]}$ given in inset (a1). Inset (a2) shows the variation of $\Delta_{1}^{[\mathrm{NT}]}$ with $N$. See legend for details.

Fig. 4 shows that $\Delta_{1}^{[N]}$ could then be viewed as a measure of the additional heat that would have been exchanged if we had retained higher-order eigenfunctions in the truncated solution.

Interestingly, for the two-term truncated solution, $N=0$, the linear system (32) and (33) can be solved to give

$\Delta_{1}^{[0]}=\frac{2(1+m k) f_{0,1}^{\prime 2}(1)+m k \Omega_{0} \lambda_{0}^{2}}{8 f_{0,1}^{\prime 2}(1)+(1+m k) \Omega_{0} \lambda_{0}^{2}}$,

which provides $A_{1}^{[0]}$ from the corresponding values of $\lambda_{0}, f_{0,1}^{\prime}(1)$, and $\Omega_{0}$. This result will be used below to account for the effect of higher-order modes in the truncated two-term solution, thereby improving its numerical accuracy.

As a final remark, we must note that in the case of balanced heat exchangers the linear system (32) and (33) should be rederived starting from the alternative series expansion (72) given in Appendix B. However, further problems are anticipated in this singular case, that will not be discussed here for brevity.

\section{Approximate two-term solution}

The gray shaded regions in the center plot of Fig. 2 indicate regions where the second lowest eigenvalue in absolute value $\left(\lambda_{-1}\right.$ for $m k>1, \lambda_{1}$ for $m k<1$ ) is larger than 25\% (light gray), 50\% (gray), and $100 \%$ (dark gray) of the absolute value of $\lambda_{0}$. Outside these regions, the approximate solution obtained by truncating the eigenfunction expansion to the first two modes

$\theta_{i}\left(\xi, y_{i}\right) \simeq A+C_{0} e^{-\lambda_{0} \xi} f_{0, i}\left(y_{i}\right)$,

is expected to give an accurate description of the temperature field away from the inlets, i.e. for $\lambda_{1}^{-1}<\xi<\xi_{L}-\left|\lambda_{-1}\right|^{-1}$. However, the difficulties introduced in the determination of the expansion coefficients $A$ and $C_{0}$ by the Nunge-Gill method $[12,13]$ motivated us to seek an alternative (approximate) procedure based on the truncated expressions for the bulk temperatures

$\theta_{m 1}(\xi) \simeq A-2 C_{0} e^{-\lambda_{0} \xi} \lambda_{0}^{-1} f_{0,1}^{\prime}(1)$,

$\theta_{m 2}(\xi) \simeq A-\frac{2}{m k} C_{0} e^{-\lambda_{0} \xi} \lambda_{0}^{-1} f_{0,1}^{\prime}(1)$, corresponding to the two-term solution, where we have used the orthogonality condition (31) with $n=q=0$, along with (17) to write $m k \int_{0}^{1} w\left(y_{2}\right) f_{0,2}\left(y_{2}\right) d y_{2}=\int_{0}^{1} w\left(y_{1}\right) f_{0,1}\left(y_{1}\right) d y_{1}=-\lambda_{0}^{-1} f_{0,1}^{\prime}(1)$.

\subsection{Zeroth-order solution}

Using the lumped inlet conditions

$\theta_{m 1}^{(0)}(0)=0, \quad \theta_{m 2}^{(0)}\left(\xi_{L}\right)=1$,

together with (39) and (40), leads to a first approximation for the expansion coefficients

$A^{(0)}=\frac{1}{1-(m k)^{-1} e^{-\lambda_{0} \xi_{L}}}, \quad C_{0}^{(0)}=\frac{\lambda_{0}}{2} \frac{A}{f_{0,1}^{\prime}(1)}$.

This provides approximate expressions for the temperature field

$\theta_{i}^{(0)}\left(\xi, y_{i}\right)=\frac{1+\left(\lambda_{0} / 2\right)\left[e^{-\lambda_{0} \xi} f_{0, i}\left(y_{i}\right) / f_{0,1}^{\prime}(1)\right]}{1-(m k)^{-1} e^{-\lambda_{0} \xi_{L}}}$

as well as the wall temperature, $\theta_{w}$, bulk temperatures, $\theta_{m 1}$ and $\theta_{m 2}$, and local heat-transfer rate, $v$, namely

$\theta_{w}^{(0)}(\xi)=\frac{1+\left(\lambda_{0} / 2\right)\left[e^{-\lambda_{0} \xi} / f_{0,1}^{\prime}(1)\right]}{1-(m k)^{-1} e^{-\lambda_{0} \xi_{L}}}$,

$\theta_{m 1}^{(0)}(\xi)=\frac{1-e^{-\lambda_{0} \xi}}{1-(m k)^{-1} e^{-\lambda_{0} \xi L}}$,

$\theta_{m 2}^{(0)}(\xi)=\frac{1-(m k)^{-1} e^{-\lambda_{0} \xi}}{1-(m k)^{-1} e^{-\lambda_{0} \xi L}}$,

$v^{(0)}(\xi)=\frac{\left(\lambda_{0} / 2\right) e^{-\lambda_{0} \xi}}{1-(m k)^{-1} e^{-\lambda_{0} \xi_{1}}}$.

Values for $\lambda_{0}$ and $f_{0,1}^{\prime}(1)$ corresponding to different values of $m$ and $k$ can be found in Table 1 . Of particular relevance for heat exchanger design are the values of the bulk temperature at the outlets, given by

$\theta_{m 1, \text { out }}^{(0)}=\frac{1-e^{-\lambda_{0} \xi_{L}}}{1-(m k)^{-1} e^{-\lambda_{0} \xi_{L}}}$,
$\theta_{m 2, \text { out }}^{(0)}=\frac{1-(m k)^{-1}}{1-(m k)^{-1} e^{-\lambda_{0} \xi_{L}}}$,

which provide useful engineering estimations in terms of $m, k$, and $\xi_{L}$, valid for moderately long heat exchangers, $\xi_{L} \gtrsim 1$. Note that as $\xi_{L} \rightarrow \infty$ these equations reduce to $\theta_{m 1, \text { out }} \simeq 1$ and $\theta_{m 2, \text { out }} \simeq 1-$ $(m k)^{-1}$ for $\lambda_{0}<0(m k>1)$, and $\theta_{m 1, \text { out }} \simeq m k$ and $\theta_{m 2, \text { out }} \simeq 0$ for $\lambda_{0}>0(m k<1)$. Note also that the outlet bulk temperatures are undetermined for $\lambda_{0}=0(m k=1)$, a singular case treated separately in Appendix A.

\subsection{First-order solution}

The accuracy of the two-term solution can be improved by replacing the lumped inlet conditions (42) by

$\theta_{m 1}^{(1)}(0)=\Delta_{1}, \quad \theta_{m 2}^{(1)}=1-\Delta_{2}$,

where we introduce the bulk temperature offsets

$\Delta_{1}=\theta_{m 2, \text { out }}^{(0)} \frac{\Delta_{1}^{[0]}}{2}, \quad \Delta_{2}=(m k)^{-1}\left[1-\theta_{m 1, \text { out }}^{(0)}\right] \frac{A_{1}^{[0]}}{2}$,

so as to mimic the effect of the neglected higher-order eigenfunctions at both ends of the heat exchanger. This particular choice of $A_{1}$ and $A_{2}$ ensures that the bulk temperature offsets add up to 
$\Delta_{1}^{[0]}$ in the limit of short heat exchangers, $\lim _{\xi_{L} \rightarrow 0} \theta_{m 1, \text { out }}^{(1)}=$ $\Delta_{1}+m k \Delta_{2}=\Delta_{1}^{[0]}$, while their ratio $\Delta_{1} /\left(m k \Delta_{2}\right)$ remains proportional to the ratio of the driving bulk temperature differences, $\theta_{m 2 \text {,out }}^{(0)} /\left[1-\theta_{m 1 \text {,out }}^{(0)}\right]$. In addition, $\Delta_{1}$ and $A_{2}$ vanish as $\xi_{L} \rightarrow \infty$. Slightly different expressions for $\Delta_{1}$ and $\Delta_{2}$ satisfying these conditions were also investigated, but none rendered significantly better approximations than those considered here.

Due to the linearity of the problem, the temperature field which satisfies the inlet conditions (51) can be written as

$\theta_{i}^{(1)}\left(\xi, y_{i}\right)=\Delta_{1}+\left[1-\Delta_{1}-\Delta_{2}\right] \theta_{i}^{(0)}\left(\xi, y_{i}\right)$,

leading to the following expressions

$\theta_{w}^{(1)}(\xi)=\Delta_{1}+\left[1-\Delta_{1}-\Delta_{2}\right] \theta_{w}^{(0)}(\xi)$

$\theta_{m 1}^{(1)}(\xi)=\Delta_{1}+\left[1-\Delta_{1}-\Delta_{2}\right] \theta_{m 1}^{(0)}(\xi)$,

$\theta_{m 2}^{(1)}(\xi)=\Delta_{1}+\left[1-\Delta_{1}-\Delta_{2}\right] \theta_{m 2}^{(0)}(\xi)$,

$v^{(1)}(\xi)=\left[1-\Delta_{1}-\Delta_{2}\right] v^{(0)}(\xi)$,

which provide significantly better approximations than (45)-(48), particularly for moderately short heat exchangers, $\xi_{L} \leqslant 1$.

It should be noted, however, that Eqs. (55) and (56) do not provide the outlet bulk temperatures directly, since they do not include the temperature offsets that take place at the outlets. Instead, we must use $\theta_{m 1, \text { out }}^{(1)}=\Delta_{1}+\left[1-\Delta_{1}-\Delta_{2}\right] \theta_{m 1, \text { out }}^{(0)}+m k \Delta_{2}$,

$\theta_{m 2, \text { out }}^{(1)}=\Delta_{1}+\left[1-\Delta_{1}-\Delta_{2}\right] \theta_{m 2, \text { out }}^{(0)}-(m k)^{-1} \Delta_{1}$,

where the last term in each expression represents the outlet bulk temperature offset written here in terms of the corresponding inlet temperature offset according to Eq. (13).

\section{Relevance to classical heat exchanger analysis}

\subsection{The $\varepsilon-N T U$ method}

The effectiveness, $\varepsilon$, of the heat exchanger is defined as the ratio of the actual rate of heat-transfer in the heat exchanger, $\left(\dot{m}_{1} c_{1}\right)\left(T_{2, \text { in }}-\right.$ $\left.T_{1, \text { in }}\right) \theta_{m 1, \text { out }}$, to the maximum possible rate of heat-transfer, $\left(\dot{m}_{i} c_{i}\right)_{\min }\left(T_{2, \text { in }}-T_{1, \text { in }}\right)$, where $\left(\dot{m}_{i} c_{i}\right)_{\min }=\dot{m}_{1} c_{1}$ for $m k>1$ and $\dot{m}_{2} c_{2}$ for $m k<1$. Accordingly, we have

$\varepsilon= \begin{cases}\theta_{m 1, \text { out }} & \text { for } m k \geqslant 1, \\ 1-\theta_{m 2, \text { out }} & \text { for } m k \leqslant 1 .\end{cases}$

In the heat exchanger literature (see e.g., Ref. [19]) it is well established that for a given type of exchanger the effectiveness depends only on two parameters, namely the ratio of the heat-capacity flow rates

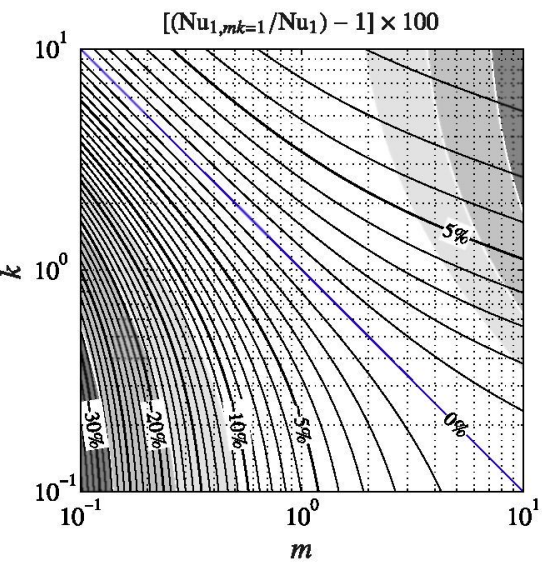

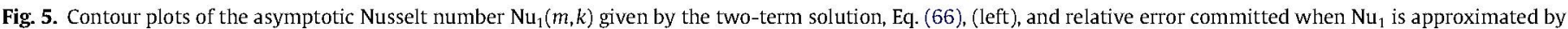

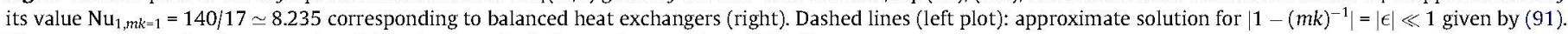
The two-term solution is expected to fail in the gray shaded areas (see caption of Fig. 2).
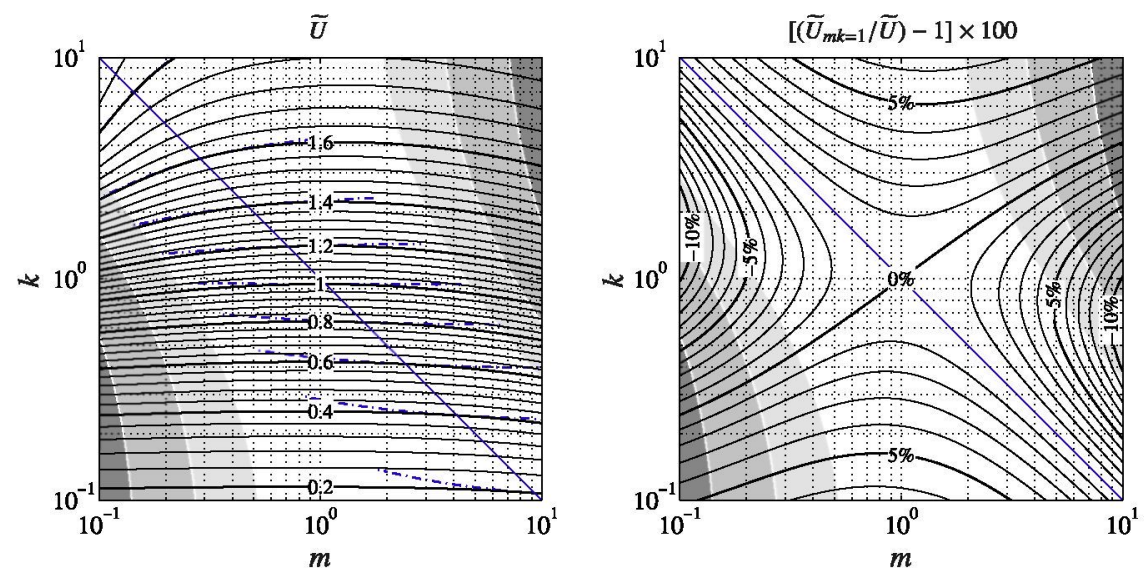

Fig. 6. Contour plots of the asymptotic overall heat-transfer coefficient $\widetilde{U}(m, k)$ given by the two-term solution, Eq. (67), (left plot), and relative error committed when $\widetilde{U}$ is approximated by its value $\widetilde{U}_{m k-1}=(35 / 17)[k /(k+1)]$ corresponding to balanced heat exchangers (right plot). Dashed lines (left plot): approximate solution for $\left|\mathbf{1}-(m k)^{-1}\right|=|\epsilon| \mid \ll \mathbf{1}$ given by (92). The two-term solution is expected to fail in the gray shaded areas (see caption of Fig. 2). 
$R=\frac{\left(\dot{m}_{i} c_{i}\right)_{\min }}{\left(\dot{m}_{i} c_{i}\right)_{\max }}= \begin{cases}(m k)^{-1} & \text { for } m k \geqslant 1, \\ m k & \text { for } m k \leqslant 1,\end{cases}$

and the so-called number of transfer units

$\mathrm{NTU}=\frac{U L}{\left(m_{i} c_{i}\right)_{\min }}= \begin{cases}2 \widetilde{U} \xi_{L} & \text { for } m k \geqslant 1, \\ 2 \widetilde{U} \xi_{L}(m k)^{-1} & \text { for } m k \leqslant 1,\end{cases}$

where

$U=\left.\frac{k_{1}}{T_{m 2}-T_{m 1}} \frac{\partial T_{1}}{\partial Y_{1}}\right|_{Y_{1}=a_{1}}=\frac{k_{1}}{a_{1}} \frac{v}{\left(\theta_{m 2}-\theta_{m 1}\right)}=\frac{k_{1}}{a_{1}} \widetilde{U}$

is the dimensional overall heat-transfer coefficient, and $L$ is the heat-transfer area, which in a two-dimensional problem such as the one considered here reduces to the heat exchanger length.

Equations expressing the effectiveness $\varepsilon$ in terms of $R$ and NTU are available for various types of heat exchangers. Thus, for the counterflow configuration we have

$\varepsilon(R, N T U)=\left\{\begin{array}{lll}\frac{1-e^{-\mathrm{NUU}(1-R)}}{1-R e^{-\mathrm{NU} U(1-R)}} & \text { for } m k \neq 1(R<1), \\ \frac{\mathrm{NTU}}{1+\mathrm{NTU}} & \text { for } m k=1(R=1),\end{array}\right.$

which, upon substitution of $\varepsilon, R$ and NTU in terms of $\theta_{m i, o u t}, m k, \xi_{L}$, and $\widetilde{U}$ using (60)-(62), reduces identically to (49) and (50) for $0<R<1$, and to (92) for $1-R \ll 1$. Then, the zeroth-order two-term solution would reduce to the $\varepsilon$-NTU method (except for the unknown value of $\widetilde{U}$ ), while the first-order two-term solution should be viewed as a more accurate approximation.

\subsection{Nusselt numbers and overall heat-transfer coefficient}

As previously discussed, a prerequisite for the application of the $\varepsilon$-NTU method is the knowledge of the overall heat-transfer coefficient, which in the thermally thin wall regime considered here is given by

$\frac{1}{\widetilde{U}}=\frac{4}{\mathrm{Nu}_{1}}+\frac{4}{k \mathrm{Nu}_{2}}$,

where $\mathrm{Nu}_{i}=h_{i}\left(4 a_{i}\right) / k_{i}$ represents the Nusselt number based on the hydraulic diameter, $4 a_{i}$, and the heat-transfer coefficient, $h_{i}$, of fluid $i$. Introducing dimensionless variables, from (38)-(40) we get

$$
\begin{aligned}
& \mathrm{Nu}_{1}=\frac{4 v}{\theta_{w}-\theta_{m 1}}=4 \widetilde{U} \frac{\theta_{m 2}-\theta_{m 1}}{\theta_{w}-\theta_{m 1}} \simeq 4\left(\frac{1}{f_{0,1}^{\prime}(1)}+\frac{2}{\lambda_{0}}\right)^{-1}, \\
& \widetilde{U}=\frac{v}{\theta_{m 2}-\theta_{m 1}} \simeq \frac{\lambda_{0} / 2}{1-(m k)^{-1}},
\end{aligned}
$$

while the symmetry conditions (26) and (27) provides $\mathrm{Nu}_{2}(m, k)=\mathrm{Nu}_{1}\left(m^{-1}, k^{-1}\right)$. We must point out that (67) and (66) are indeed independent of the expansion coefficients, and therefore should be viewed as exact asymptotic results valid for sufficiently long heat exchangers [13].

Fig. 5 shows contour plots of $\mathrm{Nu}_{1}$ given by (66). As seen in the figure, for balanced heat exchangers $\mathrm{Nu}_{1}$ takes the constant value $\mathrm{Nu}_{1, m k=1}=140 / 17 \simeq 8.235$ corresponding to a constant-heat-flux boundary condition (see Appendix A for details). On the other hand, for $m k \gg 1$, when fluid 2 has a much higher heat-capacity flow rate than fluid $1, \mathrm{Nu}_{1}$ tends to the constant-wall-temperature value,

Table 2

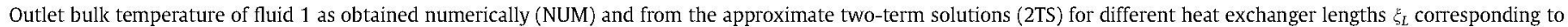

\begin{tabular}{|c|c|c|c|c|c|c|c|c|c|}
\hline$m$ & 1 & 1 & 1 & 2 & 2 & 2 & 4 & & \\
\hline$k$ & 1 & 2 & 4 & $\frac{1}{2}$ & 1 & 2 & 1 & & \\
\hline$\xi_{L}$ & & & & $\theta_{m 1, \text { out }}$ & & & & Method & Error (\%) \\
\hline \multirow[t]{4}{*}{$\frac{1}{8}$} & 0.2372 & 0.3126 & 0.3646 & 0.1843 & 0.2659 & 0.3343 & 0.2921 & NUM & \\
\hline & - & $0.3039^{a}$ & $0.3588^{\mathrm{a}}$ & - & $\underline{0.2564^{\mathrm{a}}}$ & $0.3246^{\mathrm{a}}$ & $0.2855^{\mathrm{a}}$ & 2TS exact & 3.6 \\
\hline & $0.2298^{c}$ & $0.3041^{b}$ & $0.3590^{\mathrm{b}}$ & $0.1783^{c}$ & $\underline{0.2565^{\mathrm{b}}}$ & $0.3247^{b}$ & $0.2856^{\mathrm{b}}$ & 2TS 1 st order & 3.5 \\
\hline & $0.2047^{e}$ & $0.2700^{\mathrm{d}}$ & $0.3168^{d}$ & $0.1464^{e}$ & $0.2171^{\mathrm{d}}$ & $0.2799^{\mathrm{d}}$ & 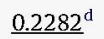 & 2TS 0th order & 22 \\
\hline \multirow[t]{4}{*}{$\frac{1}{4}$} & 0.3627 & 0.4765 & 0.5542 & 0.2859 & 0.4097 & 0.5103 & 0.4493 & NUM & \\
\hline & - & $0.4696^{a}$ & $0.5487^{\mathrm{a}}$ & - & $0.4007^{a}$ & $0.5020^{a}$ & $0.4391^{a}$ & 2TS exact & 2.3 \\
\hline & $0.3571^{c}$ & $0.4701^{b}$ & $0.5491^{b}$ & $0.2797^{c}$ & $0.4011^{\mathrm{b}}$ & $0.5023^{\mathrm{b}}$ & $\overline{0.4394^{b}}$ & 2TS 1 st order & 2.2 \\
\hline & $0.3398^{\mathrm{e}}$ & $0.4470^{d}$ & $0.5212^{d}$ & $0.2555^{\mathrm{e}}$ & $0.3722^{\mathrm{d}}$ & $0.4711^{d}$ & $\underline{0.3965^{d}}$ & 2TS 0th order & 12 \\
\hline \multirow[t]{4}{*}{$\frac{1}{2}$} & 0.5203 & 0.6761 & 0.7701 & 0.4267 & 0.5991 & 0.7247 & 0.6535 & NUM & \\
\hline & - & ${\underline{0.6646^{\mathrm{a}}}}$ & $0.7651^{\mathrm{a}}$ & - & $0.5920^{\mathrm{a}}$ & $0.7188^{a}$ & $0.6451^{\mathrm{a}}$ & 2TS exact & 1.7 \\
\hline & $0.5169^{c}$ & $0.6725^{b}$ & $0.7674^{\mathrm{b}}$ & $0.4224^{c}$ & $0.5936^{\mathrm{b}}$ & $0.7201^{b}$ & ${\underline{0.6462^{b}}}^{\mathrm{b}}$ & 2TS 1 st order & 1.1 \\
\hline & $0.5072^{\mathrm{e}}$ & $0.6602^{d}$ & $0.7541^{d}$ & $0.4070^{\mathrm{e}}$ & $0.5766^{\mathrm{d}}$ & $0.7038^{d}$ & $\underline{0.6209^{d}}$ & 2TS 0th order & 5.0 \\
\hline \multirow[t]{4}{*}{1} & 0.6790 & 0.8585 & 0.9339 & 0.5887 & 0.7950 & 0.9066 & 0.8540 & NUM & \\
\hline & - & $0.8575^{\mathrm{a}}$ & $0.9336^{\mathrm{a}}$ & - & $0.7936^{\mathrm{a}}$ & $0.9059^{\mathrm{a}}$ & ${\underline{0.8560^{\mathrm{a}}}}^{-1}$ & 2TS exact & 0.23 \\
\hline & $0.6773^{c}$ & $0.8570^{\mathrm{b}}$ & $0.9331^{\mathrm{b}}$ & $\underline{0.5863^{c}}$ & $0.7924^{\mathrm{b}}$ & $0.9051^{b}$ & $0.8509^{b}$ & 2TS 1 st order & 0.41 \\
\hline & $0.6731^{\mathrm{e}}$ & $0.8524^{d}$ & $0.9295^{d}$ & $0.5785^{\mathrm{e}}$ & $0.7850^{\mathrm{d}}$ & $0.8999^{d}$ & $0.8410^{c}$ & 2TS 0th order & 1.7 \\
\hline \multirow[t]{4}{*}{2} & 0.8068 & 0.9670 & 0.9940 & 0.7372 & 0.9359 & 0.9882 & 0.9717 & NUM & \\
\hline & - & $0.9668^{\mathrm{a}}$ & $0.9940^{\mathrm{a}}$ & - & $\underline{0.9354^{\mathrm{a}}}$ & $0.9881^{\mathrm{a}}$ & $0.9713^{\mathrm{a}}$ & 2TS exact & 0.05 \\
\hline & $0.8061^{c}$ & $0.9667^{b}$ & $0.9940^{\mathrm{b}}$ & $\underline{0.7361^{c}}$ & $\overline{0.9352^{b}}$ & $0.9881^{b}$ & $0.9711^{b}$ & 2TS 1 st order & 0.15 \\
\hline & $0.8046^{\mathrm{e}}$ & $0.9658^{d}$ & $0.9937^{d}$ & $0.7330^{\mathrm{e}}$ & $0.9332^{d}$ & $0.9874^{d}$ & $0.9693^{d}$ & 2TS 0th order & 0.6 \\
\hline \multirow[t]{4}{*}{4} & 0.8924 & 0.9979 & 0.9999 & 0.8476 & 0.9924 & 0.9998 & 0.9988 & NUM & \\
\hline & - & $0.9979^{\mathrm{a}}$ & $0.9999^{\mathrm{a}}$ & - & $\underline{0.9923}^{\mathrm{a}}$ & $0.9998^{\mathrm{a}}$ & $0.9988^{\mathrm{a}}$ & 2TS exact & 0.01 \\
\hline & $0.8922^{c}$ & $0.9979^{b}$ & $0.9999^{\mathrm{b}}$ & $\underline{0.8470}^{\mathrm{c}}$ & $\overline{0.9923^{b}}$ & $0.9998^{b}$ & $0.9988^{b}$ & 2TS 1 st order & 0.07 \\
\hline & $0.8917^{\mathrm{e}}$ & $0.9978^{d}$ & $0.9999^{\mathrm{d}}$ & $\overline{0.8459} \mathrm{v}$ & $0.9921^{d}$ & $0.9998^{d}$ & $0.9988^{d}$ & 2TS 0th order & 0.20 \\
\hline
\end{tabular}

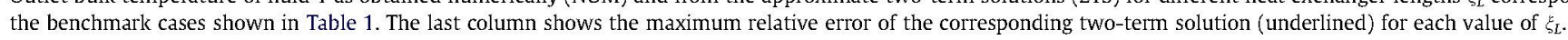

a (35) particularized for $N=0$ with $A$ and $C_{0}$ obtained from the two-term truncated system (32)-(34).

$\mathrm{b}(58)$ and (52) with $\Delta_{1}^{[0]}$ given by (37) and $\theta_{m 1 \text { out }}^{(0)}$ evaluated as indicated in footnote ${ }^{\mathrm{d}}$.

${ }^{c}(58)$ and $(52)$ with $\Delta_{1}^{[0]}$ given by $(90)$ and $\theta_{m 1,0 u t}^{(0)}$ evaluated as indicated in footnote ${ }^{\mathrm{e}}$.

d (49) with $\lambda_{0}$ and $f_{0,1}^{\prime}(1)$ obtained numerically (e.g., Table 1 ).

e (88) with $l_{1}$ and $l_{2}$ given by (73) and (77). 
$\mathrm{Nu}_{T}=7.541$ [26]. The figure also shows that, within the validity limits of the two-term solution, the constant-heat-flux boundary condition, $\mathrm{Nu}_{1} \simeq \mathrm{Nu}_{1, m k-1}$, leads to relative errors up to $10 \%$ above (for $m k>1$ ) and $20 \%$ below (for $m k<1$ ) the actual value of $\mathrm{Nu}_{1}$.

Fig. 6 shows contour plots of $\widetilde{U}$ given by (67) along with the relative error committed when $\widetilde{U}$ is approximated by the balanced heat exchanger value $\widetilde{U}_{m k=1}=(35 / 17)[k /(k+1)]$ (see Appendix A for details). Interestingly enough, the figure shows that the error committed when using the approximation $\mathrm{Nu}_{i} \simeq \mathrm{Nu}_{i, m k=1}$ tends to cancel out in (65), so that the overall error of the approximation $\widetilde{U} \simeq \widetilde{U}_{m k=1}$ turns out to be smaller than expected, particularly around the bisector line $m=k$.

\section{Numerical solution and discussion of results}

To check the accuracy of the theoretical results presented in the previous section, finite-difference numerical solutions were carried out for selected values of $m, k$, and $\xi_{L}$. Eqs. (1) and (2) were discretized using second-order centered finite differences on a distorted grid with points clustered near the inlet, outlet and separating wall. Second-order non-centered schemes were used to discretize the derivatives appearing in the boundary conditions (4)-(7). The steady solution was obtained using a time-marching method with local time stepping until the convergence criteria $|\mathcal{R}|<10^{-5}$ was achieved, $|\mathcal{R}|$ being a suitable norm of the residuals (e.g., the infinite norm) [27].

The reference case $\xi_{L}=m=k=1$ was chosen so as to assess the effect of grid resolution and check the accuracy of the numerical method. Accordingly, numerical solutions were obtained with three different levels of grid refinement: $33 \times 33,65 \times 65$, and $129 \times 129$ grid points. The numerical error of the fine grid solution was evaluated applying Richardson extrapolation to the coarse, medium, and fine grid solutions, resulting in an average error of $7.5 \times 10^{-5}$ over the interior grid points, with a maximum local
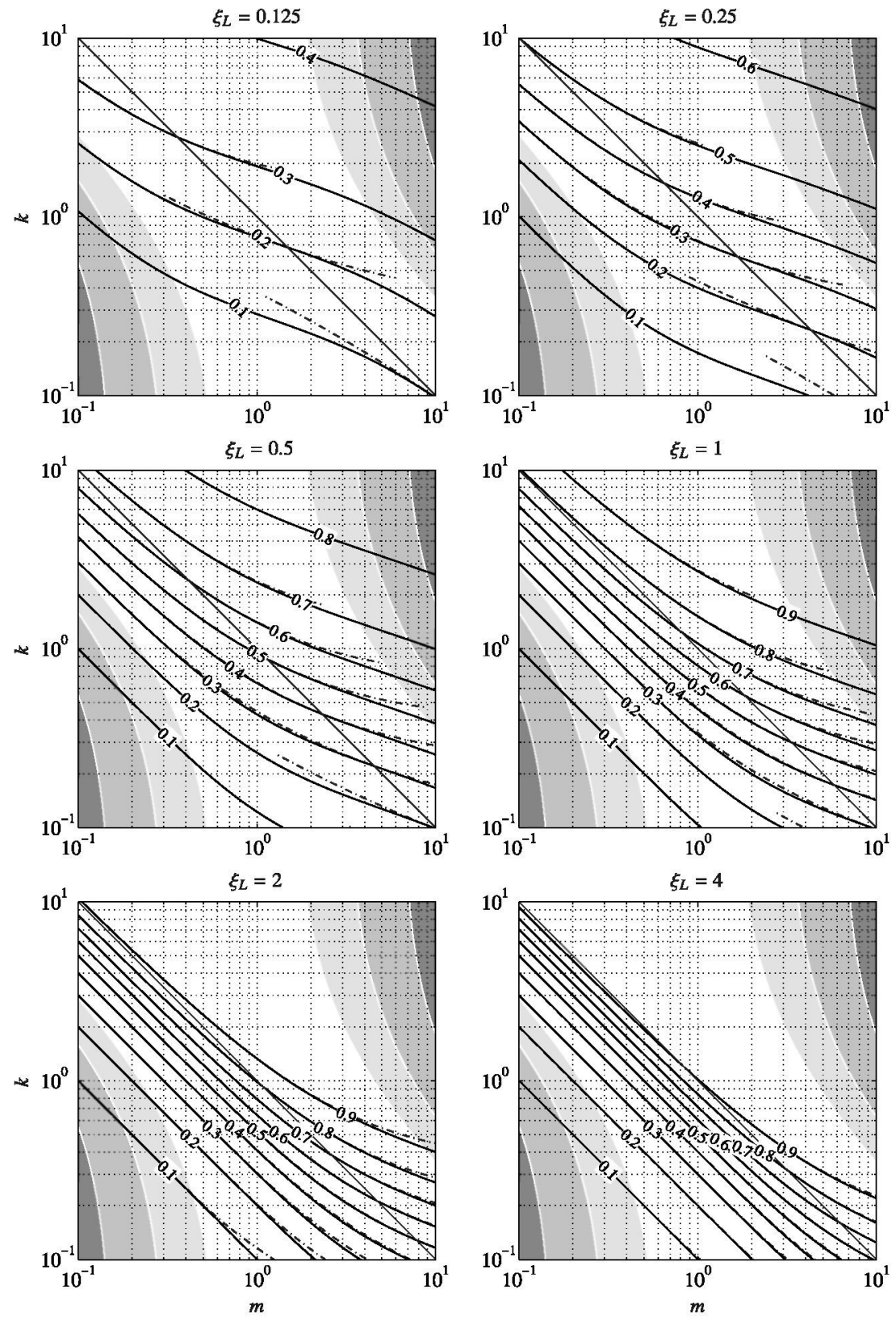

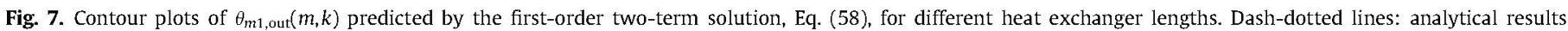

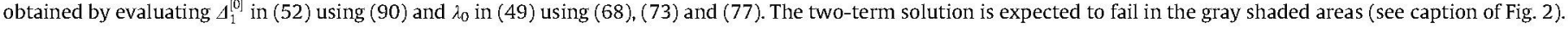


error of $6.3 \times 10^{-4}[28]$. This error estimate motivated the convergence criterium $|\mathcal{R}|<10^{-5}$ used to stop the iterative process, thus yielding a four-digit accurate solution.

Table 2 compares the values of $\theta_{m 1, \text { out }}$ calculated numerically for different values of $m, k$, and $\xi_{L}$, with the predictions of the exact, zeroth-order, and first-order two-term solutions. It also shows the maximum relative error committed by the different two-term solutions for each heat exchanger length. As can be seen, the first order solution provides the best agreement to the numerical results, specially for short heat exchangers, yielding errors less than $4 \%$ for $\xi_{L} \gtrsim 1 / 8$ and $1 \%$ for $\xi_{L} \gtrsim 1 / 2$, whereas the zeroth-order solution leads to significantly larger errors - by a factor of order 5. Note also that the two-term solutions consistently under-predict the value of $\theta_{m 1, \text { out }}$.

It is interesting to note that the different performance of the zeroth- and first-order solutions arises only from the differences in the inlet boundary conditions. Specifically, while the zerothorder solution replaces the exact inlet conditions (6) and (7) by the lumped conditions (42), the first-order solution still considers the exact inlet conditions in (93)-(96), which eventually leads to the corrected inlet conditions given by (37) and (52) (see Appendix B for details). Accordingly, the results of Table 2 show that the error introduced by using the lumped inlet conditions (42) instead of (52) is largely more important - by a factor of order 3 or $\mathbf{4}$ - than the error introduced by neglecting higher-order modes.

Fig. 7 shows contour plots of the outlet bulk temperature $\theta_{\mathrm{m} 1, \text { out }}^{(1)}$ given by the first-order two-term solution (58) together with (37), (49), (50), and (52) using the exact (i.e. numerical) values of $\lambda_{0}(m, k)$ and $A_{1}^{[0]}(m, k)$ for the different values of $\xi_{L}$ considered in Table 2. The numerical results obtained from the approximate two-term solution are plotted along with the fully analytical results obtained by using the asymptotic expressions for $\lambda_{0}$ and $A_{1}^{0]}$ corresponding to nearly-balanced exchangers, (68) and (90), in (49) and (52). As can be seen, the agreement, which is always excellent for $\left|1-(m k)^{-1}\right|=|\epsilon| \ll 1$, gets better for larger values of $|\epsilon|$ as the heat exchanger length is increased, approaching the previously discussed limit, $\theta_{m 1 \text {,out }} \simeq 1$ for $m k>1$ and $\theta_{m 1, \text { out }} \simeq m k$ for $m k<1$, as $\xi_{L} \rightarrow \infty$. As stated above, the relative error of the plotted results is expected to be smaller than a few percent even for the smaller values of $\xi_{L}$.

Fig. 8 shows the spatial variations of the wall temperature, $\theta_{w}$, mixing-cup temperatures, $\theta_{m 1}$ and $\theta_{m 2}$, local heat-transfer
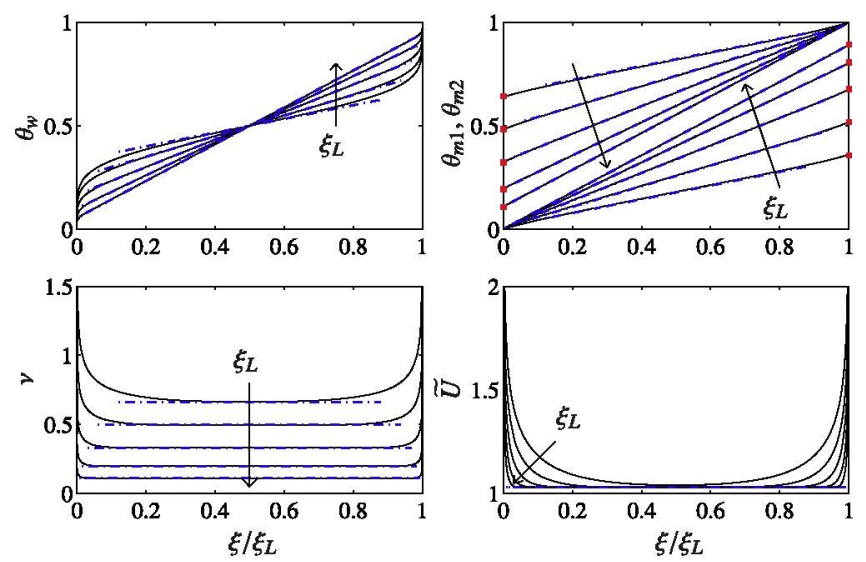

Fig. 8. Wall temperature, $\theta_{w}$, mixing-cup temperatures, $\theta_{m 1}$ and $\theta_{m 2}$, local heattransfer rate, $v$, and overall heat-transfer coefficient, $\widetilde{U}=v /\left(\theta_{m 2}-\theta_{m 1}\right)$, plotted as a function of $\xi$ for $m=k=1$ and different values of $\xi_{L}=[0.25,0.5,1,2,4]$. The arrows indicate the trends for increasing values of $\xi_{L}$. Dash-dotted lines: approximate results given by the first-order two-term solution in the limit $1-(m k)^{-1}=\epsilon=0$, Eqs. 54, 55, 56, 57 and (92) along with 52, 82, 83, 84, 85, 86, 87, and (90).
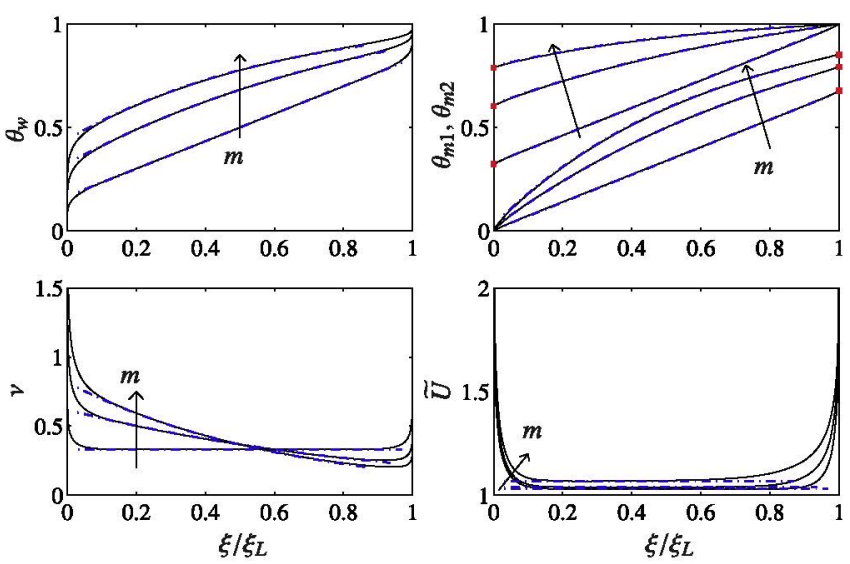

Fig. 9. Wall temperature, $\theta_{w}$, mixing-cup temperatures, $\theta_{m 1}$ and $\theta_{m 2}$, local heattransfer rate, $v$, and overall heat-transfer coefficient, $\widetilde{U}=v /\left(\theta_{m 2}-\theta_{m 1}\right)$, plotted as a function of $\xi$ for $\xi_{L}=k=1$ and different values of $m=[1,2,4]$. The arrows indicate the trends for increasing values of $m$. Dash-dotted lines: approximate results given by the first-order two-term solution, Eqs. 54, 55, 56, 57 and (67) along with (37), 45, $46,47,48$, and (52).

rate, $v$, and overall heat-transfer coefficient, $\widetilde{U}=v /\left(\theta_{m 2}-\theta_{m 1}\right)$, obtained numerically for $m=k=1$ and several values of $\xi_{L}$ ranging from short to long heat exchangers. For comparison purposes, the approximate profiles predicted by the first-order two-term solution are also plotted, showing a good agreement with the numerical results in their expected region of validity, for $\lambda_{1}^{-1}<\xi<\xi_{L}-\left|\lambda_{-1}\right|^{-1}$. In the thermal entrance regions, where the effect of the higher-order modes becomes important, the solution exhibits a singular behavior that is no longer well predicted by the approximate two-term expansion. However, it can be seen that the effect of the thermal entrance regions in the outlet bulk temperatures is appropriately accounted for by the corrections introduced in (58) and (59), which constitute excellent approximations for the outlet bulk temperatures of both streams.

Figs. 9 and 10 show similar result for $\xi_{L}=1$ and different values of $m$ and $k$, respectively. In both cases, the predictions of the two-term solution are plotted for $\lambda_{1}^{-1}<\xi<\xi_{L}-\left|\lambda_{-1}\right|^{-1}$, where and excellent agreement with the numerical results is found. Again, outlet bulk temperatures are accurately predicted by (58) and (59).
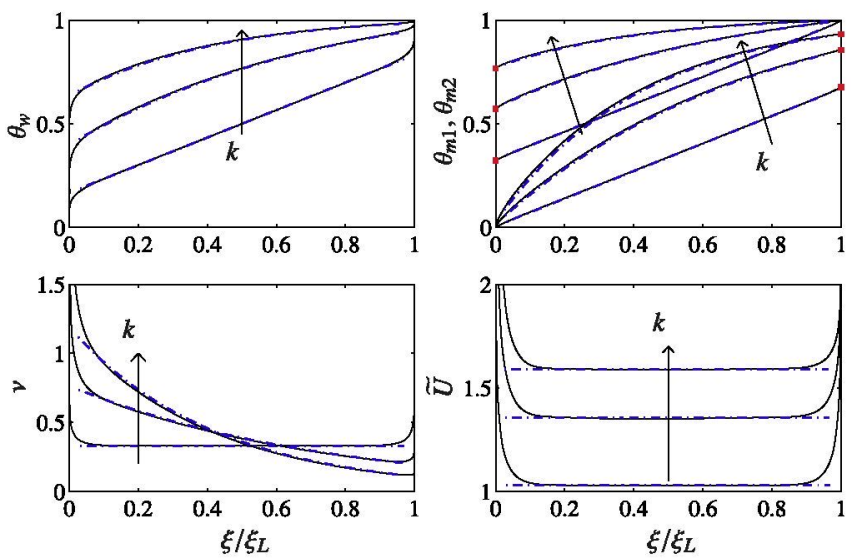

Fig. 10. Wall temperature, $\theta_{w}$, mixing-cup temperatures, $\theta_{m 1}$ and $\theta_{m 2}$, local heattransfer rate, $v$, and overall heat-transfer coefficient, $\widetilde{U}=v /\left(\theta_{m 2}-\theta_{m 1}\right)$, plotted as a function of $\xi$ for $\xi_{I}=m=1$ and different values of $k=[1,2,4]$. The arrows indicate the trends for increasing values of $k$. Dash-dotted lines: approximate results given by the first-order two-term solution, Eqs. 54, 55, 56, 57 and (67) along with (37), 45, $46,47,48$, and (52). 


\section{Conclusions}

An analytical an numerical study of multilayered, laminar, counterflow, parallel-plate heat exchangers has been presented. The analysis, valid for constant property fluids, neglects upstream conduction both in the fluids and in the plates, assumed to be thermally thin. Three dimensionless parameters emerge from the dimensionless formulation of the problem: the ratio of the Graetz numbers of both streams, $m=\left(a_{2} \mathrm{Pe}_{2}\right) /\left(a_{1} \mathrm{Pe}_{1}\right)$, the normalized heat conductivity ratio, $k=\left(a_{1} k_{2}\right) /\left(a_{2} k_{1}\right)$, and the dimensionless heat exchanger length, $\xi_{L}=L /\left(a_{1} \mathrm{Pe}_{1}\right)$.

The theoretical model was solved in terms of eigenfunction expansions involving infinite sets of both positive and negative eigenvalues [12]. Using standard symbolic algebra packages, analytical expressions for the eigenfunctions were obtained in terms of Whittaker functions, which were then used to evaluate the eigenvalues numerically. To make the calculations easier, asymptotic expressions for the eigenvalues were also derived. Contour plots of the lowest order eigenvalues and plots of the corresponding eigenfunctions were presented, along with highly precise numerical values of the eigenvalues for selected values of $m$ and $k$.

Since higher-order eigenfunctions decay exponentially fast away from the inlets, an approximate two-term solution which retains only the first non-vanishing eigenvalue was considered. The corresponding expansion coefficients were obtained from inlet conditions of different levels of complexity, leading to the exact, zeroth- and first-order two-term solutions discussed in Section 4. The two-term solutions provided analytical expressions for the outlet bulk temperatures - or, equivalently, the heat exchanger effectiveness - as well as for the spatial distributions of wall temperature, $\theta_{w}$, mixing-cup temperatures, $\theta_{m 1}$ and $\theta_{m 2}$, local heat-transfer rate, $v$, and overall heat-transfer coefficient, $\widetilde{U}$. These expressions, involving the parameters $m, k$, and $\xi_{L}$, along with the numerical values of $\lambda_{0}$, $f_{0,1}^{\prime}(1)$, and $\Omega_{0}$, were accompanied by closed form asymptotic expressions involving only the parameters $k$ and $1-(m k)^{-1}=\epsilon$ in the limit of nearly-balanced heat exchangers $|\epsilon| \ll 1$.

The analytical expressions resulting from the first-order twoterm solution were compared with finite-difference numerical simulations, showing very good agreement in terms of outlet bulk temperatures, mixing-cup and plate temperature distributions, local heat-transfer rate, overall heat-transfer coefficient, and Nusselt numbers. This suggests the possibility of using the analytical solutions presented herein as useful engineering design tools even for moderately short heat exchangers.

Future investigations could extend the present analysis to nonzero wall thermal resistances and to cylindrical geometries. In addition, the singular nature of the solution in the thermal entrance regions could be unveiled by considering the conjugate fluid-fluid problem that arises in the thermal entrance region of laminar counterflow heat exchangers.

\section{Acknowledgements}

This work was supported by Projects S-505/ENE/0229 of the Spanish Comunidad de Madrid and ENE2008-06683-C03-02 of the Spanish Ministerio de Ciencia e Innovación. MV is indebted to Drs. Antonio Acosta, Antonio L. Sánchez, and Domingo Santana from Universidad Carlos III de Madrid for helpful comments and suggestions.

\section{Appendix A. Solution for nearly-balanced heat exchangers}

\section{A.1. Asymptotic expansions for $\lambda_{0}$ and $f_{0, i}\left(y_{i}\right)$}

When the ratio of the heat-capacity flow rates of both streams is close to unity, a regular perturbation analysis leads to the following asymptotic expansions $\lambda_{0}=\epsilon l_{1}+\epsilon^{2} l_{2}+\mathcal{O}\left(\epsilon^{3}\right)$,

$f_{0, i}\left(y_{i}\right)=1+\epsilon F_{1, i}\left(y_{i}\right)+\epsilon^{2} F_{2, i}\left(y_{i}\right)+\mathcal{O}\left(\epsilon^{3}\right), \quad i=1,2$.

for the lowest eigenvalue and the corresponding eigenfunction, in terms of the small parameter $1-(m k)^{-1}=\epsilon$. Introducing (68) and (69) in (16) we may write

$$
\begin{aligned}
\theta_{i}\left(\xi, y_{i}\right)= & A+C_{0} e^{-\left[\epsilon l_{1}+\epsilon^{2} l_{2}+\mathcal{O}\left(\epsilon^{3}\right)\right] \xi}\left[1+\epsilon F_{1, i}\left(y_{i}\right)+\epsilon^{2} F_{2, i}\left(y_{i}\right)+\mathcal{O}\left(\epsilon^{3}\right)\right] \\
& +\sum_{\substack{n=-\infty \\
n \neq 0}}^{+\infty} C_{n} e^{-\lambda_{n} \xi} f_{n, i}\left(y_{i}\right)
\end{aligned}
$$

which expanding the exponential reduces to

$$
\begin{aligned}
\theta_{i}\left(\xi, y_{i}\right) \simeq & A+C_{0}+\epsilon C_{0}\left[F_{1, i}\left(y_{i}\right)-l_{1} \xi\right] \\
& +\epsilon^{2} C_{0}\left[F_{2, i}\left(y_{i}\right)-l_{2} \xi-l_{1} \xi F_{1, i}\left(y_{i}\right)+l_{1}^{2} \frac{\xi^{2}}{2}\right]+\mathcal{O}\left(\epsilon^{3} C_{0}\right) \\
& +\sum_{\substack{n=-\infty \\
n \neq 0}}^{+\infty} C_{n} e^{-\lambda_{n} \xi} f_{n, i}\left(y_{i}\right) .
\end{aligned}
$$

According to this, in the limit $\epsilon \rightarrow 0$ the solution would reduce to a constant unless we assume the scaling $\epsilon C_{0}=\mathcal{O}(1)$. Thus, in order to obtain a physically meaningful solution we must rewrite (71) as

$$
\begin{aligned}
\theta_{i}\left(\xi, y_{i}\right) \simeq \bar{A}+\bar{B}\left\{\left[F_{1, i}\left(y_{i}\right)-l_{1} \xi\right]+\epsilon\left[F_{2, i}\left(y_{i}\right)-l_{1} \xi F_{1, i}\left(y_{i}\right)-l_{2} \xi+l_{1}^{2} \frac{\xi^{2}}{2}\right]\right. \\
\left.+\mathcal{O}\left(\epsilon^{2}\right)\right\}+\sum_{\substack{n=-\infty \\
n \neq 0}}^{+\infty} C_{n} e^{-\lambda_{n} \xi} f_{n, i}\left(y_{i}\right),
\end{aligned}
$$

where both $\bar{A}=A+C_{0}$ and $\bar{B}=\epsilon C_{0}$ are $\mathcal{O}(1)$ coefficients in the limit $\epsilon \rightarrow 0$.

Rewriting $m=(1-\epsilon)^{-1} k^{-1}$ and substituting expansion (72) in Eqs. (1) and (2), in the boundary conditions (4) and (5), and in the normalization condition (21), rewritten here as $F_{k, i}(1)=0$, we obtain a hierarchy of problems that can be solved successively at different orders for the unknowns $I_{n}$ and $F_{k, i}$. Thus, to first-order, we have

$l_{1}=\frac{70}{17} \frac{k}{(k+1)}$

$F_{1,1}\left(y_{1}\right)=l_{1} \Phi_{1}\left(y_{1}\right)$

$F_{1,2}\left(y_{2}\right)=-\frac{l_{1}}{k} \Phi_{1}\left(y_{2}\right)$

$\Phi_{1}\left(y_{i}\right)=\frac{y_{i}^{4}}{16}-\frac{3 y_{i}^{2}}{8}+\frac{5}{16}$

and, to second-order,

$\frac{l_{2}}{l_{1}}=-\frac{823}{9537} \frac{(k-1)}{(k+1)}$,

$F_{2,1}\left(y_{1}\right)=\frac{l_{2}}{l_{1}} l_{1} \Phi_{1}\left(y_{1}\right)+l_{1}^{2} \Phi_{2}\left(y_{1}\right)$

$F_{2,2}\left(y_{2}\right)=-\frac{l_{1}}{k}\left(1+\frac{l_{2}}{l_{1}}\right) \Phi_{1}\left(y_{2}\right)+\frac{l_{1}^{2}}{k^{2}} \Phi_{2}\left(y_{2}\right)$,

$\Phi_{2}\left(y_{i}\right)=\frac{3 y_{i}^{8}}{3584}-\frac{7 y_{i}^{6}}{640}+\frac{11 y_{i}^{4}}{256}-\frac{15 y_{i}^{2}}{128}+\frac{1511}{17920}$

where the coefficient $l_{n}$ must be obtained by imposing the continuity of heat fluxes at the heat conducting wall at order $n+1$.

\section{A.2. Asymptotic expansion for $\Omega_{0}$}

To get an asymptotic expansion for $\Omega_{0}$ we apply formal derivation with respect to $\lambda_{n}$ to Eqs. (17) and (18) as well as the boundary 
condition (19) and normalization condition (21). Substituting the above expansions for $\lambda_{0}$ and $f_{0, i}\left(y_{i}\right)$, rewriting $m=(1-\epsilon)^{-1} k^{-1}$, and expanding the result in series of $\epsilon$, we obtain a new hierarchy of problems which can be solved to give

$\Omega_{0}=-1-\epsilon \frac{3 k-1}{2(k+1)}-\epsilon^{2} \frac{38971 k^{2}-15782 k+823}{19074(k+1)^{2}}+\mathcal{O}\left(\epsilon^{3}\right)$

\section{A.3. Heat exchanger performance}

\section{A.3.1. Zeroth-order solution}

Even though Eqs. (43)-(50) apply for any non-zero value of $\epsilon$, they exhibit a singular behavior for $\epsilon \ll 1$ that can be solved using the asymptotic expansions for $\lambda_{0}$ and $f_{0, i}\left(y_{i}\right)$ given above. This leads to asymptotic expansions for the coefficients

$$
\begin{aligned}
\bar{A}^{(0)}= & \frac{17}{70} \frac{l_{1}}{\left(1+l_{1} \xi_{L}\right)}\left\{1+\frac{\epsilon}{1+l_{1} \xi_{L}}\left[l_{1} \xi_{L}\left(1+\frac{l_{1} \xi_{L}}{2}-2 \frac{l_{2}}{l_{1}}\right)-\frac{l_{2}}{l_{1}}\right.\right. \\
& \left.\left.+\left(\frac{823}{39270} l_{1}+2 \frac{l_{2}}{l_{1}}\right)\left(1+l_{1} \xi_{L}\right)\right]+\mathcal{O}\left(\epsilon^{2}\right)\right\}, \\
\bar{B}^{(0)}= & -\frac{1}{1+l_{1} \xi_{L}}\left\{1+\frac{\epsilon}{1+l_{1} \xi_{L}}\left[l_{1} \xi_{L}\left(1+\frac{l_{1} \xi_{L}}{2}-\frac{l_{2}}{l_{1}}\right)\right.\right. \\
& \left.\left.-\frac{17}{70} l_{1}\left(1+l_{1} \xi_{L}\right)\right]+\mathcal{O}\left(\epsilon^{2}\right)\right\},
\end{aligned}
$$

wall and bulk temperature distributions

$$
\begin{aligned}
\theta_{w}^{(0)}(\xi)= & \bar{A}^{(0)}-\bar{B}^{(0)} l_{1} \xi\left\{1+\epsilon\left[\frac{l_{2}}{l_{1}}-\frac{l_{1} \xi}{2}\right]+\mathcal{O}\left(\epsilon^{2}\right)\right\}, \\
\theta_{m 1}^{(0)}(\xi)= & \bar{A}^{(0)}+\bar{B}^{(0)}\left\{\frac{17}{70} l_{1}-l_{1} \xi+\epsilon\left[\frac{17}{70} l_{1}\left(\frac{148}{561} l_{1}+\frac{l_{2}}{l_{1}}\right)\right.\right. \\
& \left.\left.-l_{1} \xi\left(\frac{17}{70} l_{1}+\frac{l_{2}}{l_{1}}-\frac{l_{1} \xi}{2}\right)\right]+\mathcal{O}\left(\epsilon^{2}\right)\right\}, \\
\theta_{m 2}^{(0)}(\xi)= & \bar{A}^{(0)}+\bar{B}^{(0)}\left\{-\frac{17}{70} \frac{l_{1}}{k}-l_{1} \xi+\epsilon\left[\frac{17}{70} \frac{l_{1}}{k}\left(\frac{148}{561} \frac{l_{1}}{k}-\frac{l_{1}+l_{2}}{l_{1}}\right)\right.\right. \\
& \left.\left.+l_{1} \xi\left(\frac{17}{70} \frac{l_{1}}{k}-\frac{l_{2}}{l_{1}}+\frac{l_{1} \xi}{2}\right)\right]+\mathcal{O}\left(\epsilon^{2}\right)\right\},
\end{aligned}
$$

local heat-transfer rate

$$
v^{(0)}(\xi)=-\bar{B}^{(0)} \frac{l_{1}}{2}\left\{1+\epsilon\left[\frac{17}{70} l_{1}+\frac{l_{2}}{l_{1}}-l_{1} \xi\right]+\mathcal{O}\left(\epsilon^{2}\right)\right\},
$$

and outlet bulk temperatures

$$
\begin{aligned}
& \theta_{m 1, \text { out }}^{(0)}=\frac{l_{1} \xi_{L}}{1+l_{1} \xi_{L}}\left\{1+\frac{\epsilon}{1+l_{1} \xi_{L}}\left[\frac{l_{2}}{l_{1}}+\frac{l_{1} \xi}{2}\right]+\mathcal{O}\left(\epsilon^{2}\right)\right\}, \\
& \theta_{m 2, \text { out }}^{(0)}=\frac{1}{1+l_{1} \xi_{L}}\left\{1+\frac{\epsilon l_{1} \xi_{L}}{1+l_{1} \xi_{L}}\left[1-\frac{l_{2}}{l_{1}}+\frac{l_{1} \xi}{2}\right]+\mathcal{O}\left(\epsilon^{2}\right)\right\} .
\end{aligned}
$$

\section{A.3.2. First-order corrections}

Approximate analytical expressions for the first-order two-term solution can be obtained by using (82)-(89) in (52)-(59). However, we also need an asymptotic expansion for $\Delta_{1}^{[0]}$ in (52), which can be obtained substituting expansions (68), (69), and (81) for $\lambda_{0}, f_{0,1}^{\prime}(1)$, and $\Omega_{0}$ in (37), eventually yielding

$$
\begin{aligned}
\mathcal{A}_{1}^{[0]}= & \frac{823\left(k^{2}+1\right)}{11183+19074 k+11183 k^{2}} \\
& +\frac{p_{0}+p_{1} k+p_{2} k^{2}+p_{3} k^{3}+p_{4} k^{4}}{13\left(11183+19074 k+11183 k^{2}\right)^{2}} \epsilon+\mathcal{O}\left(\epsilon^{2}\right),
\end{aligned}
$$

with $p_{0}=97101270, p_{1}=172721802, p_{2}=119646917, p_{3}=31350924$, and $p_{4}=22545647$. It should be noted that in the derivation of $(90)$ it was necessary to retain terms up to order $\epsilon^{4}$ in (68) and (69) and up to order $\epsilon^{3}$ in $(81)$.

\section{A.3.3. Nusselt numbers and overall heat-transfer coefficient}

Using the asymptotic expansions for $\lambda_{0}$ and $f_{0, i}\left(y_{i}\right)$ given above we get approximate expressions for the Nusselt number

$\mathrm{Nu}_{1}=\frac{140}{17}\left\{1+\epsilon\left[\frac{17}{70} l_{1}+2 \frac{l_{2}}{l_{1}}\right]+\mathcal{O}\left(\epsilon^{2}\right)\right\}$,

and overall heat-transfer coefficient

$\widetilde{U}=\frac{l_{1}}{2}\left\{1+\epsilon \frac{l_{2}}{l_{1}}+\mathcal{O}\left(\epsilon^{2}\right)\right\}$.

It is interesting to note that even though the bulk temperature differences and local heat-transfer rate are functions of $\xi$, both $\widetilde{U}$ and $\mathrm{Nu}_{1}$ are constant, up to order $\epsilon$, throughout the heat exchanger.

Results for balanced heat exchangers follow by setting $\epsilon=0$ in (82)-(92). Notice that in this case (91) reduces to the classical Nusselt number for a constant heat flux boundary condition, i.e. $\mathrm{Nu}_{1}=140 / 17 \simeq 8.235[26]$.

\section{Appendix B. Determination of the expansion coefficients}

The coefficients $A$ and $C_{n}$ appearing in (16) can be obtained following the procedure introduced by Nunge and Gill [12]. Summarizing, we take the inner product of $\theta_{i}\left(\xi, y_{i}\right)$ with the eigenfunction $f_{n}$ at both ends of the heat exchanger, $\xi=0$ and $\xi=\xi_{L}$, and use the inlet and outlet conditions

$$
\begin{aligned}
& \theta_{1}\left(0, y_{1}\right)=A+\sum_{q=-\infty}^{+\infty} C_{q} f_{q, 1}\left(y_{1}\right)=0, \\
& \theta_{2}\left(0, y_{2}\right)=A+\sum_{q=-\infty}^{+\infty} C_{q} f_{q, 2}\left(y_{2}\right)=\theta_{2, \text { out }}\left(y_{2}\right), \\
& \theta_{1}\left(\xi_{L}, y_{1}\right)=A+\sum_{q=-\infty}^{+\infty} C_{q} e^{-\lambda_{q} \xi_{L}} f_{q, 1}\left(y_{1}\right)=\theta_{1, \text { out }}\left(y_{1}\right), \\
& \theta_{2}\left(\xi_{L}, y_{2}\right)=A+\sum_{q=-\infty}^{+\infty} C_{q} e^{-\lambda_{q} \xi_{L}} f_{q, 2}\left(y_{2}\right)=1,
\end{aligned}
$$

to write

$$
\begin{aligned}
& \left\langle\theta_{i}\left(0, y_{i}\right), f_{n}\right\rangle=\left\langle A, f_{n}\right\rangle+\sum_{q=-\infty}^{+\infty} C_{q}\left\langle f_{q}, f_{n}\right\rangle, \\
& \left\langle\theta_{i}\left(\xi_{L}, y_{i}\right), f_{n}\right\rangle=\left\langle A, f_{n}\right\rangle+\sum_{q=-\infty}^{+\infty} C_{q} e^{-\lambda_{q} \xi_{L}}\left\langle f_{q}, f_{n}\right\rangle .
\end{aligned}
$$

According to the orthogonality conditions (29)-(31) the inner products $\left\langle A, f_{n}\right\rangle$ and $\left\langle f_{q}, f_{n}\right\rangle$, for $q \neq n$, are identically zero, thus we have

$C_{n}=\frac{\left\langle\theta_{i}\left(0, y_{i}\right), f_{n}\right\rangle}{\left\langle f_{n}, f_{n}\right\rangle}=\frac{e^{\lambda_{n} \xi_{L}}\left\langle\theta_{i}\left(\xi_{L}, y_{i}\right), f_{n}\right\rangle}{\left\langle f_{n}, f_{n}\right\rangle}$

Equating the numerators of the above expressions for $C_{n}$ and using (93)-(95) to substitute $\theta_{i}\left(0, y_{i}\right)$ and $\theta_{i}\left(\xi_{L}, y_{i}\right)$ we get

$$
\begin{aligned}
& -m k \int_{0}^{1} w\left(y_{2}\right) \theta_{2, \text { out }}\left(y_{2}\right) f_{n, 2}\left(y_{2}\right) d y_{2} \\
& =e^{\lambda_{n} t_{L}}\left[\int_{0}^{1} w\left(y_{1}\right) \theta_{1, \text { out }}\left(y_{1}\right) f_{n, 1}\left(y_{1}\right) d y_{1}-m k \int_{0}^{1} w\left(y_{2}\right) f_{n, 2}\left(y_{2}\right) d y_{2}\right] .
\end{aligned}
$$


Expanding $\theta_{i, \text { out }}\left(y_{i}\right)$ in series using (94) and (95) and applying standard simplifying techniques based on (17)-(21) it is possible to obtain analytical expressions for the resulting integrals, which leads to Eq. (33). Substituting $f_{n}$ by 1 and $\lambda_{n}$ by 0 and operating similarly we obtain Eq. (32).

\section{References}

[1] S. Hardt, W. Ehrfeld, V. Hessel, Strategies for size reduction of microreactors by heat transfer enhancement effects, Chem. Eng. Commun. 190 (2003) 540-559.

[2] J. Esarte, G. Min, D.M. Rowe, Modelling heat exchangers for thermoelectric generators, J. Power Sources 93 (2001) 72-76.

[3] ]. Yu, H. Zhao, A numerical model for thermoelectric generator with the parallel-plate heat exchanger, J. Power Sources 172 (2007) 428-434.

[4] R.S. Wakeland, R.M. Keoliana, Effectiveness of parallel-plate heat exchangers in thermoacoustic devices, J. Acoust. Soc. Am. 115 (2004) 288-2873.

[5] E.D. Marquardt, R. Radebaugh, Compact High Effectiveness Parallel Plate Heat Exchangers, in: R.G. Ross Jr. (Ed.), Cryocoolers, vol. 12, Kluwer Academic/ Plenum Publishers, 2003, pp. 507-516.

[6] G.F. Nellis, A heat exchanger model that includes axial conduction, parasitic heat loads, and property variations, Cryogenics 43 (2003) 523-538.

[7] R. Radebaugh, Microscale heat transfer at low temperatures, in: $S$. Kakaç et al. (Eds.), Microscale Heat Transfer - Fundamentals and Applications, Springer, 2005, pp. 93-124.

[8] R.K. Shah, A.L. London, Laminar Flow Forced Convection in Ducts: A Source Book for Compact Heat Exchanger Analytical Data, Academic Press, New York, 1978. pp. 196-207.

[9] T.L. Perelman, On conjugated problems of heat transfer, Int. J. Heat Mass Transfer 3 (1961) 293-303.

[10] E. Papoutsakis, D. Ramkrishna, Conjugated Graetz problems. I. General formalism and a class of solid-fluid problems, Chem. Eng. Sci. 36 (1981) 1381-1390.

[11] E. Papoutsakis, D. Ramkrishna, Conjugated Graetz problems. II. Fluid-fluid problems, Chem. Eng. Sci. 36 (1981) 1393-1399.
[12] RJ. Nunge, W.N. Gill, Analysis of heat or mass transfer in some countercurrent flows, Int. J. Heat Mass Transfer 8 (1965) 873-886.

[13] R.J. Nunge, W.N. Gill, An analytical study of laminar counterflow double-pipe heat exchangers, AIChE J. 12 (1966) 279-289.

[14] S. Mori, M. Kataya, A. Tanimoto, Performance of counterflow, parallel plate heat exchangers under laminar flow conditions, Heat Transfer Eng. 2 (1980) 28-38.

[15] P.J. Schmid, D.S. Henningson, Stability and Transition in Shear Flows, Applied Math. Sciences, vol. 142, Springer-Verlag, New York, 2001.

[16] S.N. Singh, Heat transfer by laminar flow in a cylindrical tube, Appl. Sci. Res. A7 (1958) 325-340.

[17] R. Nunge, E.W. Porta, W.N. Gill, Axial conduction in the fluid streams of multistream heat exchangers, Chem. Eng. Progr. Symp. Series 63 (1967) 80-91.

[18] W.M. Kays, A.L. London, Compact Heat Exchangers, 3rd ed., McGraw-Hill, New York, 1984.

[19] F.P. Incropera, D.P. DeWitt, T.L. Bergman, A.S. Lavine, Fundamentals of Heat and Mass Transfer, sixth ed., John Wiley \& Sons, 2007.

[20] F. Scofano Neto, R.M. Cotta, Counterflow double-pipe heat exchanger analysis using a mixed lumped-differential formulation, Int. J. Heat Mass Transfer 35 (1992) 1723-1731.

[21] E. Whittaker, G. Watson, Modern Analysis, Camb. Univ. Pres., 1952.

[22] M. Abramowitz, l. Stegun (Eds.), Handbook of Mathematical Functions with Formulas, Graphs, and Mathematical Tables, Chapter 13, National Bureau of Standards, vol. 55, Applied Mathematics Series, 1964.

[23] H.A. Lauwerier, The use of confluent hypergeometric functions in mathematical physics and the solution of an eigenvalue problem, Appl. Sci. Res. A2 (1951) 184-204.

[24] A. Erdêlyi, C.A. Swanson, Asymptotic forms of Whittaker's confluent hypergeometric functions, Mem. Am. Math. Soc. 25 (1957) 1-49.

[25] A. Zettl, Sturm-Liouville Theory, Mathematical Surveys and Monographs, vol. 121, American Mathematical Societys, 2005.

[26] S. Kakac, R.K. Shah, W. Aung, Handbook of Single-Phase Convective Heat Transfer, John Wiley and Sons, Inc., New York, 1987.

[27] C.A.J. Fletcher, Computational Techniques for Fluid Dynamics, Springer Series in Computational Physics, vols. I and II, Springer, 1988.

[28] P.J. Roache, Verification and Validation in Computational Science and Engineering, Hermosa Publishers, Albuquerque, New Mexico, 1998. 